\title{
A Continuum Dislocation Dynamics Crystal Plasticity Approach to Irradiated BCC $\alpha$-Iron
}

\author{
Stephanie A. Pitts* \\ Wen Jiang \\ Computational Mechanics \& Materials \\ Idaho National Laboratory \\ Idaho Falls, Idaho 83415 \\ Email: stephanie.pitts@inl.gov
}

Davide Pizzocri

Department of Energy, Nuclear Reactors Group

Politecnico di Milano

20156 Milano, Italy

Erin I. Barker

Applied Statistics \& Computational Modeling

Pacific Northwest National Laboratory

Richland, WA, 99352

Hussein M. Zbib

Mechanical and Materials Engineering

Washington State University

Pullman, WA 99164

\section{ABSTRACT}

Radiation-induced embrittlement of reactor pressure vessel (RPV) steels can potentially limit the operating life of nuclear power plants. Over extended exposure to radiation doses, these body-centered cubic (BCC) irons 
to capture the interaction among dislocations and self-interstitial atom (SIA) loops in $\alpha$-iron. We demonstrate the importance of modeling cross slip using a combined stochastic Monte Carlo approach and the role of slip system strength anisotropy in capturing stochastic cross slip interactions. Through these captured interactions, the CDD crystal plasticity model can capture both the stress response and the physical evolution of dislocation on different slip system planes. Single-crystal verification experiments are used to calibrate the CDD crystal plasticity model, and a set of simplified polycrystalline simulations demonstrates the model's ability to capture the stress response from tensile experiments on $\alpha$-iron.

\section{Introduction}

Ferritic-martensitic irons are used in the reactor pressure vessels (RPVs) of existing light water reactor nuclear power plants, due to their resistance to irradiation damage [1]. These materials are also under consideration for some advanced small modular reactor designs [2]. Despite this resistance, over extended exposure to radiation doses, these body-centered cubic (BCC) irons demonstrate irradiation damage, the primary radiation damage defect being self-interstitial atom (SIA) loops [3]. These irradiation defects interact with dislocations, increasing the brittleness of the material by impeding the movement of dislocations $[4,5]$. The impeded dislocation motion produces increased yield stress and significant hardening in the plastic regime, causing the material to behave in a brittle manner [2]. In addition to significant radiation loads, the RPV is subjected to thermal and pressure loading from power plant operation. The combination of these effects causes the degradation of the RPV over time as impeded dislocation movement in the microstructure reduces the ability of the material to accommodate mechanical loading [6]. RPVs must have sufficient toughness to avoid fracture during normal operation or transient events, and embrittlement reduces material toughness over time.

Crystal plasticity models play a key role in capturing how mesoscale changes in the microscale affect the engineering scale material properties. Recent developments in crystal plasticity modeling have pushed towards models based on dislocation density [7, 8]. These models, developed for face-centered cubic (FCC) materials, use dislocation density evolution equations with origins in the simple generation and annihilation terms proposed by Kocks et al. [9]. These models separate out populations of screw and edge dislocations, yet the evolution equations for the dislocation populations still reduce to a binary multiplication and annihilation balance. Also working with FCC materials, Roters et al. and Ma et al. used the concept of mobile and immobile dislocations in their dislocation evolution models [10,11].

Radiation damage has been incorporated into FCC crystal plasticity models through dispersed barrier models [12,13]; in these models the radiation damage defect density is held constant. Newer crystal plasticity models focused on RPV applications couple irradiation defects to the dislocation evolution model by adding to the slip resistance calculation [14-17]. Among these crystal plasticity models that couple dislocations and SIA loop evolution, two employ a dislocation evolution model that tracks both mobile and immobile dislocations. These dislocation evolution models include terms for observable dislocation interaction mechanisms, including locking and cross slip [15,17]. The implemented cross slip term, however, relies on an approach that can saturate the dislocation density and does not account for the stochastic nature of cross slip 
Copyright within the physical crystal microstructure.

Several groups have focused on the problem of modeling the interactions among dislocations and SIA loops in BCC metals, yet none have fully captured the physical interactions among key microstructural components, particularly in regard to cross slip dislocations. Since this work focuses on capturing the physical interactions among dislocations and SIA loops, we performed simulations using $\alpha$-iron exclusively.

In this work, we present a dislocation-density-based continuum crystal plasticity model composed of physically based interaction mechanisms in the dislocation evolution equations, including a stochastic approach to cross slip calculations in order to capture the random nature of this dislocation mechanism. The dislocation evolution model is coupled to an equation for SIA loop evolution, and hardening is calculated as a function of both dislocation and SIA interactions. Single-crystal verification experiments are used to calibrate the continuum dislocation dynamics (CDD) crystal plasticity model prior to assessing the model's predictive capability using a simplified polycrystalline geometry. In Section 2, we introduce the constitutive model as well as details on the dislocation and SIA loop evolution equations. In Section 3, we describe our calibration of the CDD crystal plasticity model to single-crystal $\alpha$-iron tensile experiments. In Section 4 , we present the results of the finite element simulations, including a discussion of cross slip approaches and a comparison to lower length scale approaches.

\section{Model Details}

As done by [18], our crystal plasticity model uses the multiplicative decomposition of the deformation gradient into elastic and plastic components:

$$
\mathbf{F}=\mathbf{F}^{e} \mathbf{F}^{p}
$$

The crystal shape alteration due to the dislocation motion is accounted for by the plastic deformation gradient tensor, $\mathbf{F}^{p}$, while the elastic deformation gradient tensor, $\mathbf{F}^{e}$, accounts for recoverable elastic stretch and rotation of the crystal lattice. The evolution of the plastic deformation is given as:

$$
\dot{\mathbf{F}}^{p}=\mathbf{L}^{p} \mathbf{F}^{p}
$$

where $\mathbf{L}^{p}$ is the plastic velocity gradient. The plastic velocity gradient is defined as the sum of the slip increments resulting from dislocation motion on all the slip systems [18]:

$$
\mathbf{L}^{\mathbf{p}}=\sum_{\alpha=1}^{n} \dot{\gamma}^{\alpha} \mathbf{s}_{o}^{\alpha} \otimes \mathbf{m}_{o}^{\alpha}
$$


Copyright where $^{\text {by }}$ is the slip rate due to dislocation glide and $\mathbf{s}_{o}$ and $\mathbf{m}_{o}$ are the slip direction and slip plane normal unit vectors, respectively, in the reference configuration. The slip rate is connected to the glide of mobile dislocations via Orowan's relation [19]:

$$
\dot{\gamma}^{\alpha}=\rho_{m o b i l e}^{\alpha} b v_{\text {glide }}^{\alpha}
$$

where $\rho_{\text {mobile }}^{\alpha}$ is the mobile dislocation density on each slip system $\alpha, b$ is the Burgers vector of the crystal, and $v_{\text {glide }}^{\alpha}$ is the glide velocity of the mobile dislocations. The evolution of the mobile dislocation rate is a constitutive expression described in Section 2.1 below. In this work, we apply a power law expression [20] for the dislocation glide velocity:

$$
v_{\text {glide }}^{\alpha}=v_{o} b\left|\frac{\tau^{\alpha}}{g^{\alpha}}\right|^{1 / m} \operatorname{sgn}\left(\tau^{\alpha}\right) \text { if } \tau^{\alpha} \geq g_{o}^{\alpha}
$$

where $v_{o}$ is the initial dislocation velocity, $\tau^{\alpha}$ is the applied resolved shear stress on each slip system $\alpha$, and $g^{\alpha}$ is the slip system resistance or strength, further defined in Eqn. 6. The second Piola-Kirchoff stress tensor is used to calculate the applied shear stress, in a manner consistent with the use of the slip direction and slip plane normal unit vectors in Eqn. 3.

The slip system resistance, $g^{\alpha}$ in Eqn. 5, provides a measure of the strength of each slip system to resist dislocation motion. Physically-based frameworks write the constitutive slip system resistance equation as a function of dislocation and defect densities within the crystal [21,22]; both defects and other dislocations act as barriers to dislocation motion [23, 24]. In this model the resistance of the slip systems to dislocation motion is considered as the additive sum of the physical barriers to dislocation motion, including dislocation forests, and irradiation defects.

$$
g^{(\alpha)}=g_{o}^{(\alpha)}+g_{\text {forest }}^{(\alpha)}+g_{\text {irradiation-defects }}^{(\alpha)}
$$

In Eqn. 6 the initial lattice friction is represented by a constant, $g_{o}^{\alpha}$. The effect of anisotropy is accounted for by adjusting the initial slip system resistance for each type of slip system type [25]:

$$
g_{o}^{(\alpha)}= \begin{cases}2.5 \cdot g_{p s} & g_{o}^{\{110\} \text { type }} \\ 1.0 \cdot g_{p s} & g_{o}^{\{112\} t y p e} \\ 2.0 \cdot g_{p s} & g_{o}^{\{123\} t y p e}\end{cases}
$$

where $g_{p s}$ is the isotropic Peierls strength of the material. For the lower temperature case in which only the $\{110\}$ and $\{112\}$ 
Copyright planes are active [26,27], we consider only these two slip plane types in our crystal plasticity model.

The slip system resistance due to dislocation-forest-type accumulation on all slip systems is modeled with a modified Bailey-Hirsch approach [21]:

$$
g_{\text {forest }}^{(\alpha)}=\alpha_{m b h} b \mu \sqrt{\sum_{\beta} \Omega^{(\alpha \beta)}\left(\rho_{\text {mobile }}^{(\beta)}+\rho_{\text {immobile }}^{(\beta)}\right)}
$$

where the hardening is considered self-hardening when $\alpha=\beta$ and latent-hardening when $\alpha \neq \beta$. The coefficient $\alpha_{m b h}$ is a fitting parameter, $\mu$ is the shear modulus of the material, and $\Omega$ is the interaction matrix containing the self- and latenthardening parameters.

\subsection{Dislocation Evolution}

Our crystal plasticity model is based on the CDD framework [28,29], with separate terms used to describe each of the specific physical interaction mechanisms in the dislocation evolution rate terms. The mobile dislocation evolution rate is fully coupled to the immobile dislocation evolution rate. The mobile dislocation evolution is governed by six terms. Each term in the equation represents a specific physical dislocation-interaction mechanism.

$$
\begin{aligned}
\dot{\rho}_{\text {mobile }}^{(\alpha)}= & \dot{\rho}_{\text {generation }}^{(\alpha)}-\dot{\rho}_{\text {mobile-annihilation }}^{(\alpha)}-\dot{\rho}_{\text {locking }}^{(\alpha)} \\
& +\dot{\rho}_{\text {freed }}^{(\alpha)}+\dot{\rho}_{\text {cross-slip }}^{(\alpha)}-\dot{\rho}_{\text {immobile-annihilation }}^{(\alpha)}
\end{aligned}
$$

The mobile dislocation evolution equation is coupled directly to the immobile dislocation evolution rate via three of the dislocation mechanism terms:

$$
\dot{\rho}_{\text {immobile }}^{(\alpha)}=\dot{\rho}_{\text {locking }}^{(\alpha)}-\dot{\rho}_{\text {freed }}^{(\alpha)}-\dot{\rho}_{\text {immobile-annihilation }}^{(\alpha)}
$$

The primary generation term for the mobile dislocation evolution rate, Eqn. 9, is the creation of new mobile dislocations from Frank-Read sources:

$$
\dot{\rho}_{\text {generation }}^{(\alpha)}=\alpha_{1} \rho_{\text {mobile }}^{(\alpha)} v_{\text {glide }} l_{\text {inv }}
$$

The annihilation of two mobile dislocations of opposite sign is the second largest contributor to the mobile dislocation evolution rate. The annihilation interaction is allowed to occur only within a capture radius, $R_{c}$, which is defined as a factor 


$$
\dot{\rho}_{\text {mobile-annihilation }}^{(\alpha)}=\alpha_{2}\left(\rho_{\text {mobile }}^{(\alpha)}\right)^{2} 2 R_{c} b v_{\text {glide }}
$$

The locking dislocations term, which is negative in the mobile dislocation evolution (Eqn. 9) acts as the source term for the immobile dislocation evolution, Eqn. 10. This term represents the mobile dislocations that are then prevented from further movement by the dislocation locks.

$$
\dot{\rho}_{\text {locking }}^{(\alpha)}=\alpha_{3} \rho_{\text {mobile }}^{(\alpha)} v_{\text {glide }} l_{\text {inv }}
$$

The mobile and immobile dislocation evolution rates are also coupled via the freed dislocation mechanism term. This term represents those locked dislocations that are freed through the application of increasing shear stress on the slip system, and it transfers dislocations from the immobile to the mobile densities. The increasing shear stress supplies the additional driving force needed to overcome the strength of the locked barrier.

$$
\dot{\rho}_{\text {freed }}^{(\alpha)}=\alpha_{4} \rho_{\text {imm }}^{(\alpha)}\left|\frac{\tau^{(\alpha)}}{g^{(\alpha)}}\right|^{2} v_{\text {glide }} l_{\text {inv }}
$$

The cross-slip term represents the movement of mobile dislocations from one slip system to another, within the same cross slip family. Depending on the applied shear stress on the receiving slip system, these cross slip dislocations may contribute to the mobile or immobile dislocation density.

$$
\dot{\rho}_{\text {cross-slip }}^{(\alpha)}=\alpha_{5} \rho_{\text {cross-slipped }}^{(\alpha)} v_{\text {glide }} l_{\text {inv }}
$$

The final mechanism term represents the annihilation between a mobile dislocation and an immobile dislocation of opposite sign. Unlike the mobile-mobile dislocation annihilation in Eqn. 12, this term contributes to the coupling between the mobile and immobile dislocation evolution rates by removing equal quantities from both dislocation density populations.

$$
\dot{\rho}_{\text {immobile-annihilation }}^{(\alpha)}=\alpha_{6} \rho_{\text {mobile }}^{(\alpha)} \rho_{\text {imm }}^{(\alpha)} 2 R_{c} b v_{\text {glide }}
$$

In these expressions, the leading $\alpha$ coefficients are fitting parameters with values taken from dislocation dynamics simulation 
Copyright results [28], $v_{\text {glide }}$ is the dislocation glide velocity from Eqn. 5, $R_{c}$ is the radius of capture for annihilating dislocations of opposite sign, and $l_{i n v}$ is the inverse mean free glide path. The inverse mean glide path is a measure of the distance between existing dislocations through which a mobile dislocation can glide [21]:

$$
l_{\text {inv }}^{(\alpha)}=\beta_{\text {forest }} \sqrt{\sum_{\beta}\left(\rho_{\text {mobile }}^{(\beta)}+\rho_{\text {immobile }}^{(\beta)}\right)}
$$

This mean free glide path quantity represents an inhibiting force on the dislocation motion in the evolution expressions in Eqns. 11, 13, 14, and 15, representing the increased difficulty of mobile dislocation glide as the total number of dislocations within a grain accumulate.

\subsection{Dislocation Cross Slip Models}

Cross slip is a significant component of dislocation motion in BCC materials [30]. In lower length scale models such as dislocation dynamics, the probability of cross slip from single slip systems within a cross-slip family is calculated using a stochastic approach. Rhee et al. calculated the probability of cross slip as a function of applied shear stress and temperature [31].

$$
P^{\alpha \beta}=\exp \left(\frac{-\left(\tau^{*}-\left|\tau^{(\beta)}\right|\right) V_{a}}{k T}\right)
$$

where $\tau^{*}$ is a critical stress for a dislocation to cross slip (in mm), $\tau^{(\beta)}$ is the applied shear stress on a slip system $\beta$ (in mm), $V_{a}$ is the volume $\left(\mathrm{mm}^{3}\right)$ required for a dislocation to cross slip, $k$ is the Boltzmann constant, and $T$ is the temperature (in $\mathrm{K}$ ). The probability of cross slip to move to a slip system is calculated for each slip system within a cross-slip family. Cross slip systems with higher applied stresses are more likely to receive cross slip dislocations.

The challenge in continuum level models is to adapt the discrete probability model for continuum dislocation density values while retaining the physical foundation of the cross slip model. This work explores the effects of two different models for stochastic cross-slip representation within a continuum framework: a stochastic-only model and a stochastic Monte Carlo combination model for calculating dislocation cross slip. The stochastic-only model is taken from Patra and McDowell [15] and denoted in this work as 'stochastic-PM'; the combination stochastic Monte Carlo method introduced in this work is given the notation 'stochastic-MC.'

\subsubsection{Stochastic-PM Cross Slip Approach}

The stochastic-only cross slip approach is included in this work for comparison with the stochastic Monte Carlo combination approach. The stochastic-only model was implemented following the approach of Patra and McDowell [15]. In 
Copyright this approacn, the probability of cross slip is also calculated using Eqn. 18. The probability values are used directly as the coefficients for the mobile dislocation densities in order to calculate the cross slip dislocation density:

$$
\rho_{\text {cross-slipped }}^{(\alpha)}=P^{\alpha \beta} \sum_{\beta \neq \alpha}^{\left.\beta\right|_{\text {family }}} \rho_{\text {mobile }}^{(\beta)}-\rho_{\text {mobile }}^{(\alpha)} \sum_{\beta \neq \alpha}^{\left.\beta\right|_{\text {family }}} P^{\alpha \beta}
$$

where $\alpha$ represents the current slip system, $\beta$ represents the other slip systems in the same cross slip family, and $\left.\beta\right|_{\text {family }}$ is the total number of slip systems within a cross slip family. The net change of cross slipped dislocations for a single slip system depends on two values: that of the probability of cross slip, and that of the mobile dislocation densities.

\subsubsection{Stochastic-MC Cross Slip Approach}

In the stochastic Monte Carlo combination approach to modeling dislocation cross slip, we calculate the probability of cross slip via the same function used by Rhee et al., Eqn. 18 [31]. To perform the Monte-Carlo-type analysis, we construct a continuous distribution function (CDF) for each cross slip system by normalizing the probability by the sum of all probabilities within the slip system, such that the CDF is bounded between 0 and 1. A larger CDF bin indicates a correspondingly larger probability that a certain system will receive cross slipped dislocations. In the Monte Carlo analysis, performed for each slip system $\alpha$ in a cross slip family, a randomly generated number is compared against the bins in the $\mathrm{CDF}$. The bin in which the random number falls is considered the slip system $\beta$ that receives the cross slipped dislocations from slip system $\alpha$ :

$$
\rho_{\text {cross-slipped }}^{(\alpha)}=\sum_{\beta=1}^{\beta||_{\text {family }}} P^{\beta \alpha} \rho^{(\beta)}=\sum_{\beta=1}^{\left.\beta\right|_{\text {family }}} \rho_{\text {received }}^{(\beta)}-\rho_{\text {given }}^{(\alpha)}
$$

where $\left.\beta\right|_{\text {family }}$ is the total number of slip systems within a cross slip family, $\rho_{\text {received }}^{(\beta)}$ is cross slip dislocations received from other slip systems in the same cross slip family, and $\rho_{\text {given }}^{(\alpha)}$ is the amount of dislocation that cross slip from the current $(\alpha)$ system to another system. $\rho_{\text {received }}^{(\beta)}$ is only nonzero if the Monte Carlo analysis determines that the current slip system $(\alpha)$ receives cross slip dislocations from a $(\beta)$ system. If the random number generated its corresponding CDF bin are associated with the same slip system, then that slip system is considered to not produce any cross slipped dislocations, and $\rho_{\text {given }}^{(\alpha)}$ is zero.

Within the stochastic-PM approach, Eqn. 19, all slip systems participate in each cross slip transfer of dislocations: each slip system loses dislocations and gains cross slip dislocations from all other slip systems in the cross slip family at every simulation timestep. In contrast, in the stochastic-MC approach, Eqn. 20, only one slip system in the cross slip family receives all the dislocations cross slipping from the first slip system, for a single material integration point at the simulation timestep. 


\section{Copyright 2.3 Irradiation Defect Evolution}

Irradiation defects contribute to the embrittlement of the slip systems by raising the slip system resistance, denoted as $g_{\text {irradiation-defects }}^{(\alpha)}$ in Eqn. 6. In $\alpha$-iron, SIA loops act as barriers to dislocation motion, and the 3-D nature of these loops is accounted for in the barrier model with the cube root term:

$$
g_{\text {irradiation-defects }}^{(\alpha)}=\alpha_{S I A} \mu\left(\frac{b^{2} d_{S I A}}{l_{\text {SIA }}^{3}}\right)^{1 / 2}
$$

where

$$
l_{S I A}=\frac{1}{\sqrt[3]{\rho_{S I A}}}
$$

SIA loops can also be absorbed by dislocations that pass through the same plane on which an SIA loop exists. Following Mastorakos et al., the evolution of SIA loops is considered via a simple interaction model similar to the dislocation annihilation terms in Eqns. 12 and 16 [32]:

$$
\dot{\rho}_{S I A}=-4 \beta_{S I A} R_{S I A} \rho_{S I A} \sum_{\alpha} \rho_{\text {mobile }}^{(\alpha)} v_{\text {glide }}^{(\alpha)}
$$

where $\beta_{S I A}$ is a fitting parameter for SIA loop annihilation, $R_{S I A}$ is the radius within which an SIA loop can capture and absorb dislocations, and $\rho_{S I A}$ is the density of SIA loops. After an initial exposure to radiation, the density of SIA loops will decrease under mechanical loading of the crystal, as the dislocations absorb the SIA loops during the glide motion.

As with dislocation loops, the SIA loops contribute to the shortening of the mean free glide path for mobile dislocations. We account for the contribution of the SIA loops to the mean free glide path by modifying Eqn. 17 to be a function of both forest dislocations and SIA loops.

$$
l_{\text {inv }}^{(\alpha)}=\beta_{\text {forest }} \sqrt{\sum_{\beta}\left(\rho_{\text {mobile }}^{(\beta)}+\rho_{\text {immobile }}^{(\beta)}\right)}+\beta_{\text {SIA }} \sqrt{\rho_{\text {SIA }} d_{S I A}}
$$

As in Eqn. 17, $\beta_{S I A}$ is a fitting coefficient. Here, we account for the impact of the 3-D SIA loops by multiplying the density, $\rho_{S I A}$, by the average diameter, $d_{S I A}$, on the 2-D glide slip planes of the mobile dislocations. 


\section{Copyright $3{ }^{2}$ Miodel Calibration}

This crystal plasticity model has been implemented in the Multiphysics Object Oriented Software Environment (MOOSE), which was developed at Idaho National Laboratory to solve coupled physics simulations in a finite element method framework [33]. We calibrated our proposed crystal plasticity model by focusing first on unirradiated $\alpha$-iron single-crystal data before verifying the implementation of the irradiation-defect-hardening models against lower length scale simulations.

Table 1: Values of the parameters used in the crystal plasticity model for single-crystal $\alpha$-iron simulations

\begin{tabular}{ccl}
\hline Parameter & Value & Description \\
\hline$C_{11}$ & $242 \times 10^{3} \mathrm{MPa}$ & Elastic constant [23] \\
$C_{12}$ & $150 \times 10^{3} \mathrm{MPa}$ & Elastic constant [23] \\
$C_{44}$ & $112 \times 10^{3} \mathrm{MPa}$ & Elastic constant [23] \\
$\mu$ & $80 \times 10^{3} \mathrm{MPa}$ & Shear modulus [23] \\
$b$ & $2.48 \times 10^{-7} \mathrm{~mm}$ & Burgers vector [23] \\
$\dot{\gamma}_{o}$ & $4.0 \times 10^{-2}$ & Reference strain rate \\
$m$ & 0.012 & Strain rate exponent \\
$\alpha_{m b h}$ & 0.4 & Dispersed barrier \\
$\Omega^{\alpha \alpha}$ & 1.0 & Self-hardening \\
$\Omega^{\alpha \beta}$ & 0.2 & Latent-hardening \\
$R_{c}$ & $15 b \mathrm{~mm}$ & Radius of capture \\
$\tau^{*}$ & $4 \times 10^{-3} \cdot \mu$ & Critical cross slip stress \\
$V_{a}$ & $20 b^{3}$ & Cross slip volume \\
$k$ & $1.38065 \times 10^{-20}$ & Bolztmann constant \\
$T$ & $298 \mathrm{~K}$ & Temperature \\
\hline
\end{tabular}

Using single-crystal tension test experimental data [34], we calibrated the coefficient parameters for the six mechanism interaction terms used in the dislocation evolution models, Eqns. 9 and 10, against $\alpha$-iron single-crystal data from three different loading directions [35]. These particular loading orientations change the influence of the cross slip term $\left(\alpha_{5}\right.$ in Eqn.

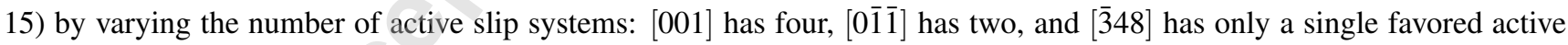
slip system.

The single-crystal calibration simulations were performed on a $1 \mathrm{~mm}^{3}$ cube mesh consisting of 2168 -node hexahedron elements. A mesh convergence study was performed, in all three loading directions shown in Fig. 1, with a series of 8 , 64, 216, and 512 elements. The loading direction with the greatest number of active slip systems, [100], demonstrated no mesh dependence while the $[\overline{3} 48]$ loading direction demonstrated the largest mesh dependence. The $[0 \overline{1} \overline{1}]$ and $[\overline{3} 48]$ loading directions demonstrate acceptable mesh convergence with 216 elements. Symmetric boundary conditions were used, and a displacement loading rate corresponding to a strain rate of $3.3 \times 10^{-4} 1 / \mathrm{s}$ was applied, matching Keh [34]. The values of 


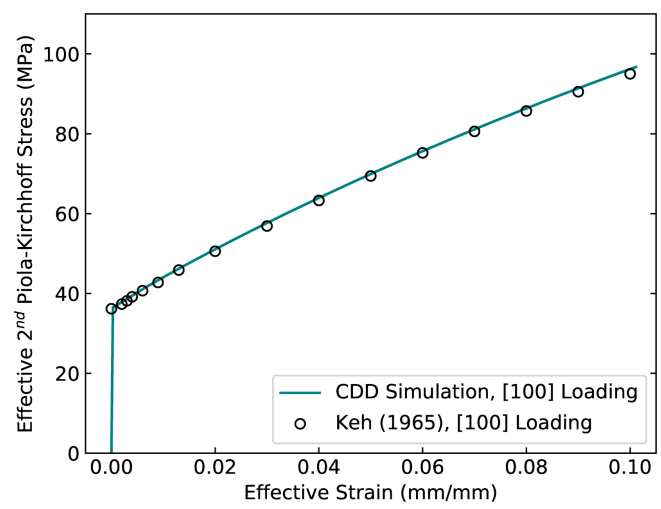

(a) Verification in the [100] loading direction of the dislocation-mechanism term $\alpha$ coefficients against single-crystal BCC $\alpha$-iron

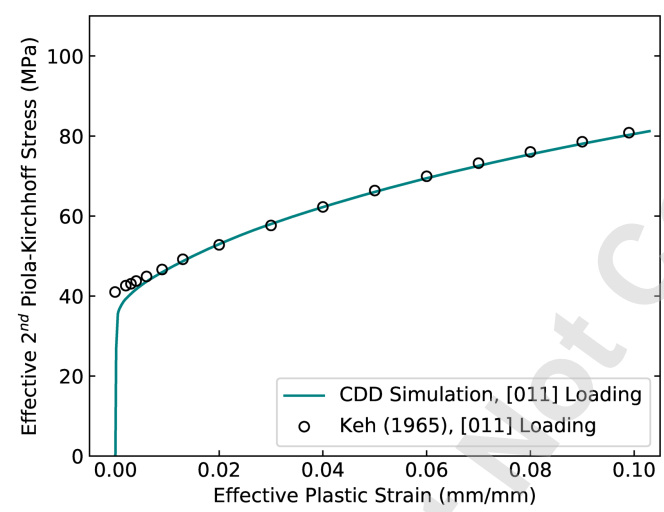

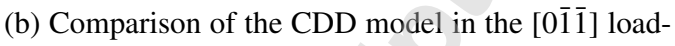
ing direction using the $\alpha$ values given in Tab. 2 with single-crystal BCC $\alpha$-iron

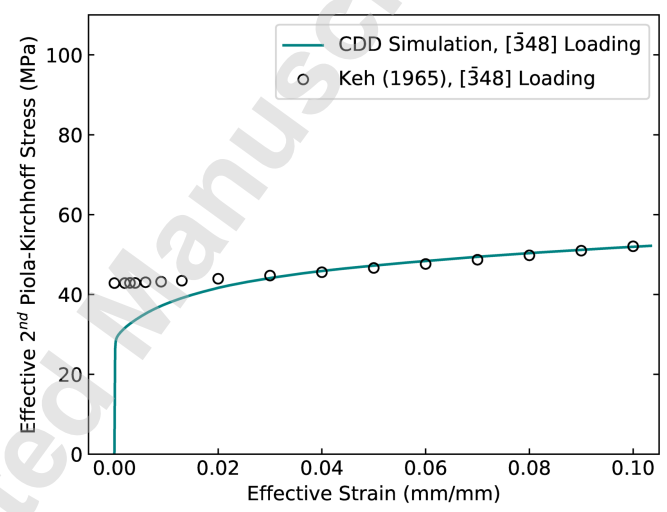

(c) Demonstration in the [348] loading direction of the dislocation evolution mechanisms $\alpha$ coefficients against single-crystal $\mathrm{BCC} \alpha$-iron

Fig. 1: Verification of the dislocation mechanism term coefficients in Eqns. 11-16 against single-crystal BCC $\alpha$-iron in three loading direction [34], reproduced from Lee et al. [35]. The misalignment at low strains of the CDD model with the

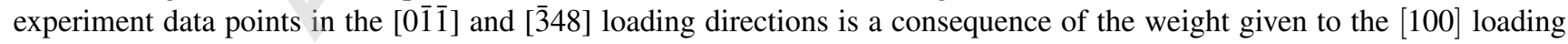
direction in setting the uniform values for the parameters $\alpha_{1}-\alpha_{4}$ and $\alpha_{6}$ across all three loading directions.

the elastic properties, glide velocity, and dislocation cross slip were held constant throughout this calibration process and are listed in Tab. 1. We assumed equal values for both the initial mobile dislocation density and the initial immobile dislocation density, set at $2.5 \times 10^{5} \mathrm{~mm}^{-2}$ [35] and distributed evenly across all slip systems. 
Copyright Table 2: Disfocation evolution parameters for Eqns. 9 and 10, calibrated for the crystal plasticity model in single-crystal $\alpha$-iron simulations for three different loading directions

\begin{tabular}{llll}
\hline \multicolumn{4}{c}{ Loading Direction } \\
& {$[100]$} & {$[0 \overline{1} \overline{1}]$} & {$[\overline{3} 48]$} \\
\hline$\alpha_{1}$ & 0.03 & 0.03 & 0.03 \\
$\alpha_{2}$ & 0.5 & 0.5 & 0.5 \\
$\alpha_{3}$ & 0.002 & 0.002 & 0.002 \\
$\alpha_{4}$ & 0.002 & 0.002 & 0.002 \\
$\alpha_{5}$ & 0.015 & 0.0335 & 0.044 \\
$\alpha_{6}$ & 1.0 & 1.0 & 1.0 \\
$g_{o}$ & $15 \mathrm{MPa}$ & $8.8 \mathrm{MPa}$ & $8.8 \mathrm{MPa}$ \\
\hline
\end{tabular}

The values of the dislocation mechanism terms, $\alpha_{1}-\alpha_{6}$, were fit using insights deduced from dislocation dynamics simulations [28]. Based on the results of these dislocation dynamics simulations, we gave priority in the fitting to the first two terms in the mobile dislocation evolution rate, Eqns. 11 and 12, and adjusted the values of the coefficients to obtain agreement with the experimental curves for each loading orientation. The crystal plasticity simulations using the finalized parameter values are presented in Fig. 1 and compared against the experimental data from Keh [34]. The specific values of the dislocation evolution parameters are listed in Tab. 2. The different Peierls stress values for the different loading directions was also observed by Taheri and Zbib [29]. The variance of the cross slip evolution parameter indicates that the role of cross slip is more significant in the deformation of the single active slip system loading direction compared to the loading direction with multiple active slip systems, as shown previously by Li et al. [28].

\section{Discussion and Results}

Inclusion of a cross slip term in the dislocation evolution equations is key for capturing the stress-strain behavior of the single slip loading orientation. Cross slip of dislocations away from the activated slip system mitigates the growth of this slip systems' dislocation density; thus, cross slip prevents over-hardening of the effective stress response.

\subsection{Stochastic-MC and Stochastic-PM Comparison}

A key feature of our crystal plasticity model is the inclusion of the combination stochastic-MC cross slip term, Eqn. 20, to help capture the random nature of the physical cross slip dislocation movement. Both the stochastic-MC approach and the more commonly applied stochastic-PM approach use the same calculation for the probability of cross slip, Eqn. 18, which is a function of the applied shear stress. The difference between the two approaches lies in the method of determining which slip systems will interact during cross slip. Based on previous studies [28], we anticipated that the largest difference in the cross slip approaches would occur in the [348] loading direction. In our comparison of single-crystal simulations, we applied 


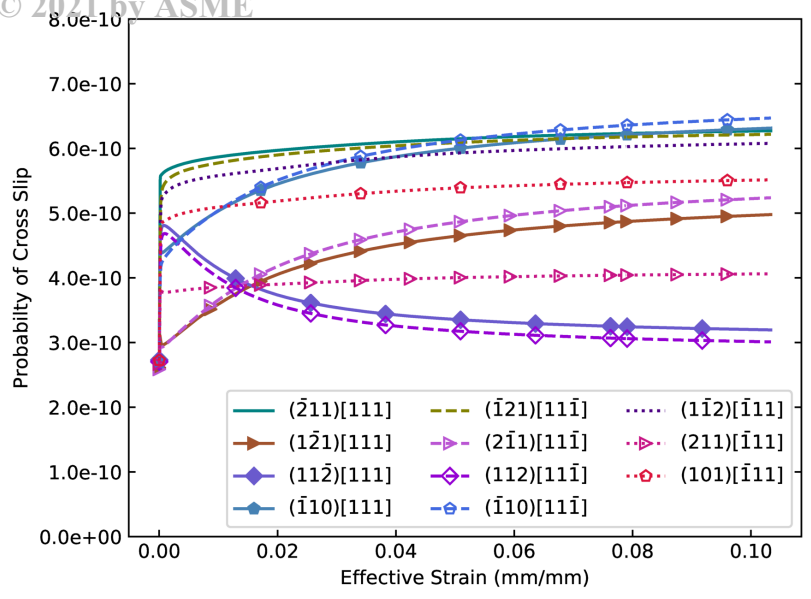

(a) Stochastic-PM approach

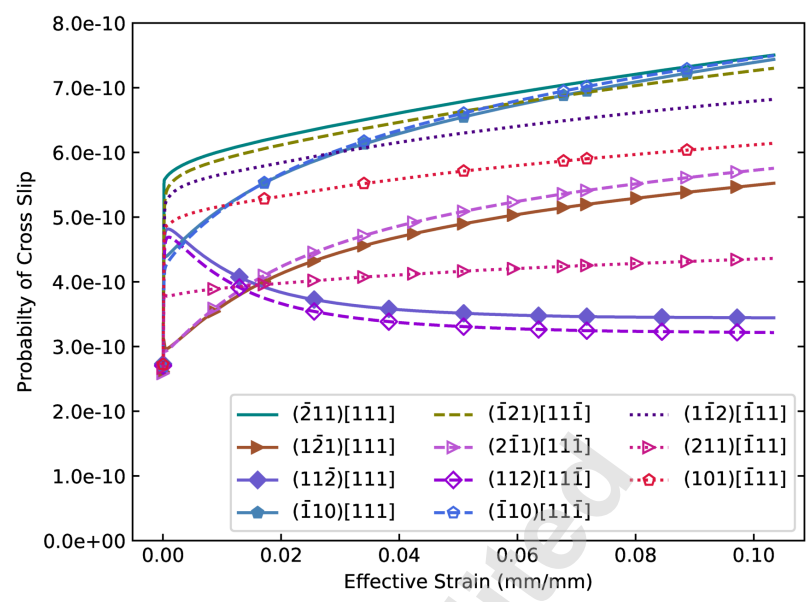

(b) Stochastic-MC approach

Fig. 2: Probability of dislocation cross slip on slip systems with an applied shear stress above the initial lattice friction strength, as calculated directly from Eqn. 18, before normalization. The stochastic-PM approach demonstrates the saturation trends shown in the mobile dislocation evolution, Fig. 4a, while the stochastic-MC approach demonstrates increased probability with increasing strain, Fig. 4 b.

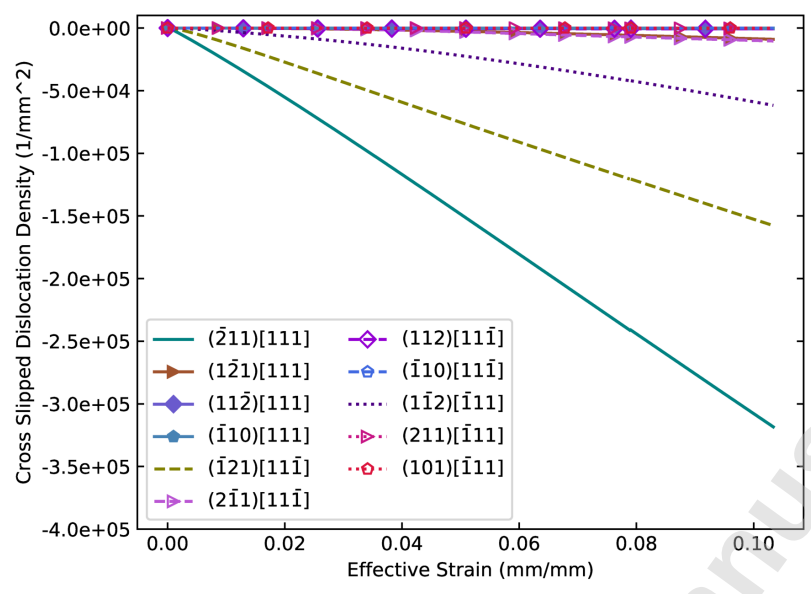

(a) Stochastic-PM approach from Eqn. 19

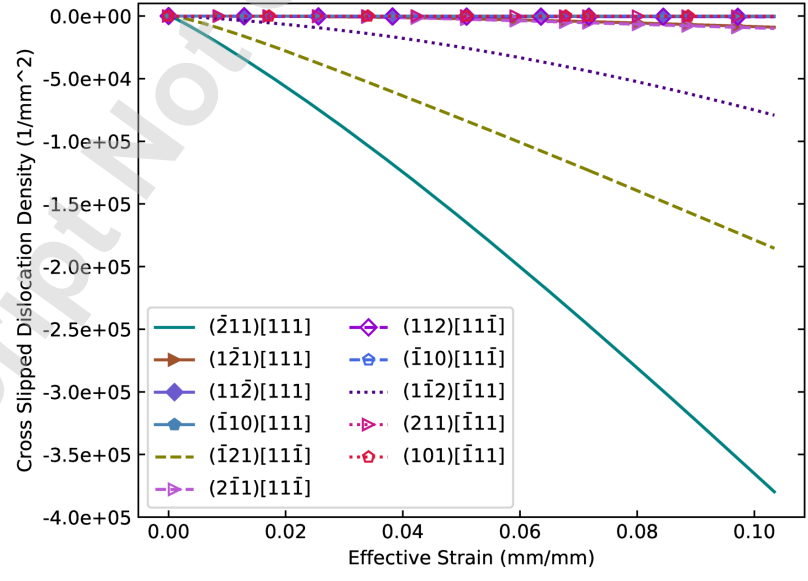

(b) Stochastic-MC approach, as calculated with Eqn. 20

Fig. 3: Evolution of the cross slip dislocation density. Note that the (211)[111] system is expected to be the favored active slip system under the [348] loading direction [34]. The value of the $\alpha_{5}$ coefficient in the stochastic-PM approach was adjusted to create parity the in $(\overline{2} 11)[111]$ cross slip dislocation at the beginning of the simulation, although the mobile dislocation evolution, Fig. 4, impacts the available dislocations density to cross slip.

the same symmetric boundary conditions and loading rate, with the material parameters given in Tabs. 1 and 2 , for the [348] loading direction. In the stochastic-PM cross slip approach, we adjust the leading coefficient, $\alpha_{5}$, to produce parity in the density of the cross slip dislocations in the two approaches at the beginning of the simulations. With these consistent trends in the probability of cross slip among slip systems, Fig. 2, we examined the impact of the two cross slip approaches on the mobile dislocation density, Fig. 3. In both approaches, Figs. 3a and 3b, the cross slip of dislocations away from the active $(\overline{2} 11)[111]$ system—as well as, to a lesser extent, away from the secondary activated $(\overline{1} 21)[11 \overline{1}]$ and $(\overline{1} 21)[11 \overline{1}]$ systems-relieves the growth of dislocations on the active slip systems.

The stochastic-PM approach, Fig. 4a, introduces an apparent saturation limit in the (211)[111] system mobile dislocation 


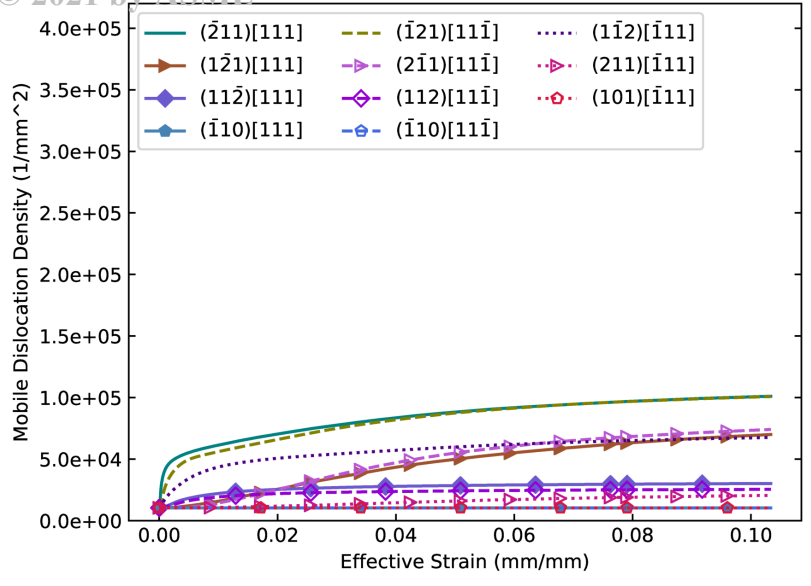

(a) The stochastic-PM approach demonstrates saturation

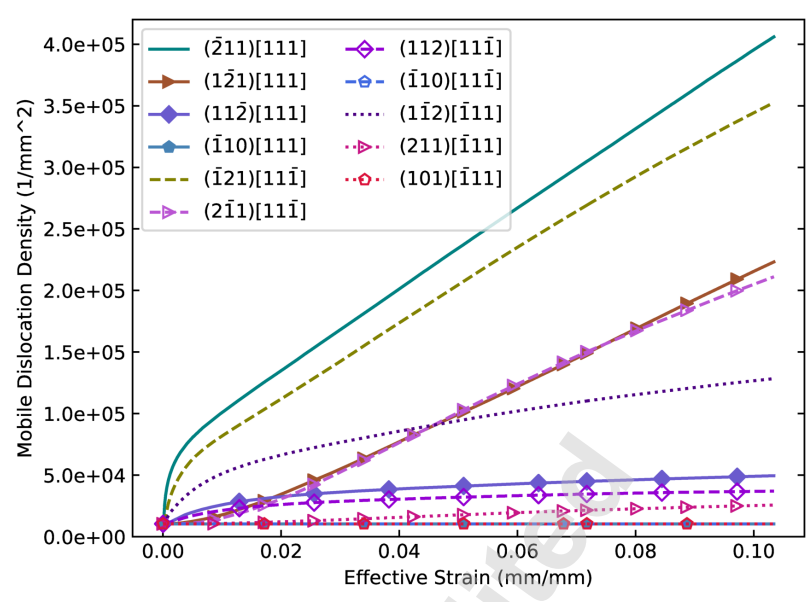

(b) The stochastic-MC approach demonstrates growth

Fig. 4: Evolution of the mobile dislocation density on select slip systems, with the $(\overline{2} 11)[111]$ system expected to be the favored active slip system [34]. The stochastic-PM approach demonstrates saturation of the mobile dislocation densities; in contrast, the stochastic-MC cross slip approach is able to maintain dislocation density growth on this primary slip system. Although other slip systems demonstrate growth as a result of slip system activation, the $(\overline{2} 11)[111]$ system shows the highest density growth in accordance with its role as the primary slip system.

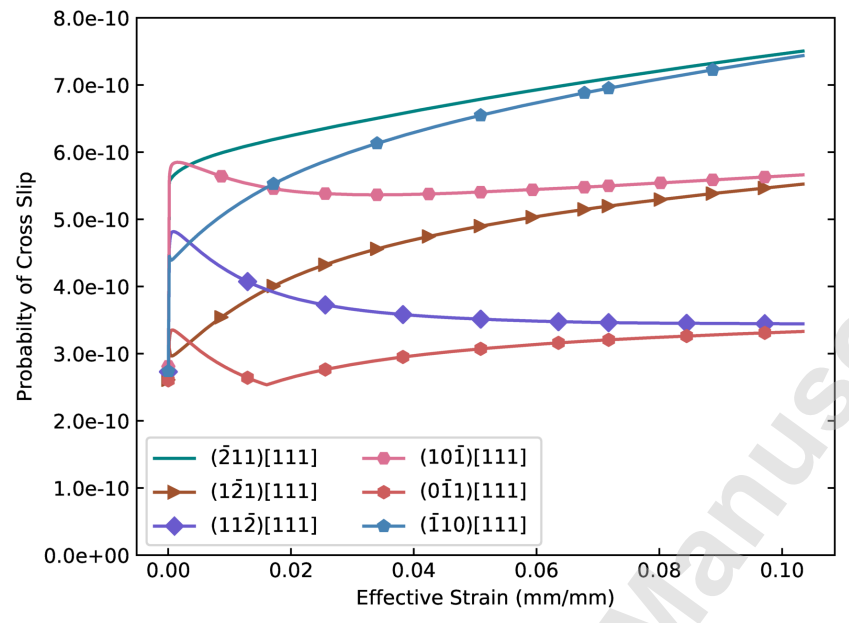

(a) Probability of cross slip in the [111] cross slip family

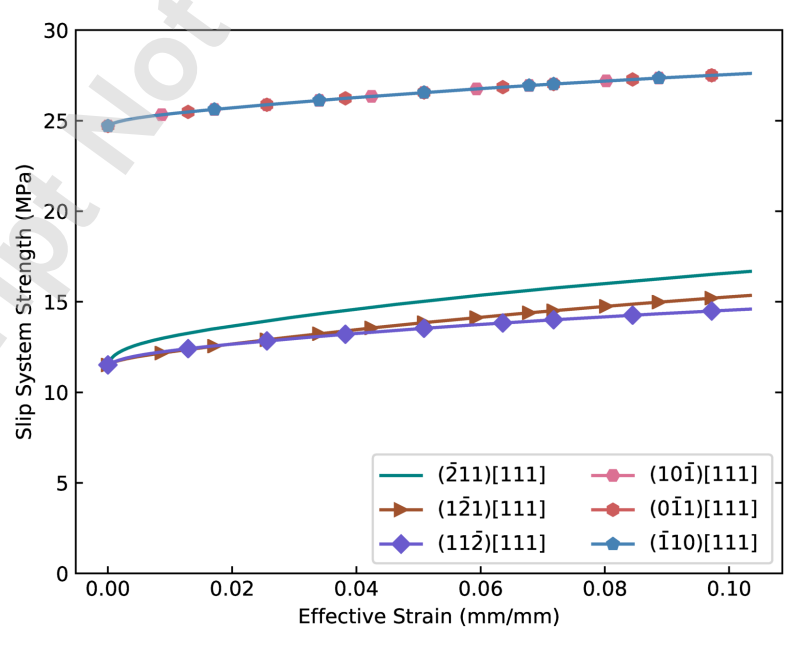

(b) Slip system strength evolution in the [111] cross slip family

Fig. 5: The comparison of the cross slip probability among the six slip systems in the [111] cross slip family demonstrates that the $(\overline{2} 11)[111]$ and $(\overline{101})[111]$ systems have similar probabilities as the strain increases. Using the stochastic-MC approach, the slip system evolution demonstrates the influence of the anistropy correction for the intrinsic lattice friction strength, Eqn. 7. This anisotropy correction hardens the $\{110\}$-type systems more than it does the $\{112\}$-type systems, enabling dislocations to cross slip from the lower slip resistance systems to the higher ones, without additional slip system activation.

density. The saturation from the deterministic approach reduces the mobile dislocation density of the $(\overline{2} 11)[111]$ system to such an extent that the mobile dislocation density of the secondary $(\overline{1} 21)[11 \overline{1}]$ system grows to parity with that of the primary $(\overline{2} 11)[111]$ system. This saturation of the $(\overline{2} 11)[111]$ system's mobile dislocation density runs counter to the expected continued dislocation density growth on the primary activated slip system. While the stochastic-PM approach does produce a stress-strain curve similar to the experimentally measured curve, as indicated by Fig. 4a, the inability of this approach to maintain the $(\overline{2} 11)[111]$ system as the primary activated slip system demonstrates the unsuitability of the stochastic-PM 
In contrast, the stochastic-MC approach, Fig. 4b, this transfer of dislocations via cross slip enables the dislocation density on the active $(\overline{2} 11)[111]$ system to grow at a moderate pace. Even though the mobile dislocation density on additional

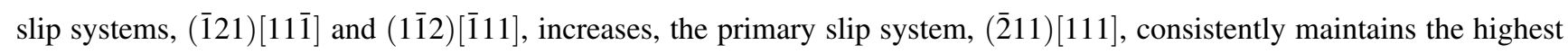
dislocation density value, as is expected in a single slip loading orientation.

\subsection{Connection between Cross Slip and Anisotropy}

Inclusion of anisotropy is necessary to capture the single slip system behavior of the [348] loading direction orientation. Similar cross slip probabilities are calculated on both the $(\overline{2} 11)[111]$ system and the $(\overline{1} 01)[111]$ system, yet the anistory of the slip systems, Eqn. 7, allows mobile dislocation growth only on the softer 112 systems. Since these two systems have the highest probability of receiving cross slip dislocations within the [111] cross slip family, it is therefore likely that dislocations will cross from $(\overline{2} 11)[111]$ to $(\overline{1} 01)[111]$ and $(\overline{1} 10)[111]$, as shown in Fig. 5a. Transfer of dislocations from the active $(\overline{2} 11)[111]$ system to the (101)[111] system relieves the dislocation growth on the active system; this dislocation growth mitigation prevents over-hardening of the crystal stress response. The interaction between the anisotropy and the stochastic-MC cross slip approach captures the single active slip system behavior expected under [348] loading.

The higher slip system resistance allows the (101)[111] and (110)[111] systems to absorb the cross slip dislocations from the $(\overline{2} 11)[111]$ system. This absorption of cross slip dislocations from an active system by an inactive system enables our crystal plasticity model to capture the near-ideal plastic behavior of the single crystal loaded in the [ $\overline{3} 48]$ direction.

\subsection{SIA Loop Evolution Verification}

Verification of the SIA loop defect model consists of comparing the stress-strain curves generated by our crystal plasticity model to those from dislocation dynamics simulations $[16,36]$. As with the dislocation dynamics simulations, these crystal plasticity simulations were performed on $\mu \mathrm{m}^{3}$ cubes. These cubes were loaded in tension in the [100] direction, with traction-free boundary conditions on the lateral sides. In alignment with the dislocation dynamics simulations, a displacement loading rate equivalent to a strain rate of $1001 / \mathrm{s}$ was applied to the top of the cubes. Six different verification simulations were run, each with a different initial SIA loop density: $1.63 \times 10^{13} \mathrm{~mm}^{-3}, 8.15 \times 10^{12} \mathrm{~mm}^{-3}, 3.61 \times 10^{12}$ $\mathrm{mm}^{-3}, 1.63 \times 10^{12} \mathrm{~mm}^{-3}, 8.15 \times 10^{11} \mathrm{~mm}^{-3}$, and unirradiated. As per Barton et al. [16], an initial dislocation density of $2 \times 10^{-7} \mathrm{~mm}^{-2}$ was assumed (split evenly between mobile and immobile dislocations), and a Peierls stress of $80 \mathrm{MPa}$ was applied. The other SIA-loop-specific parameters are given in Tab. 3. The remainder of the model constants used in these verification simulations are given in Tab. 1, and the dislocation evolution parameters for the [100] loading direction are listed in Tab. 2.

The results of these simulations compare reasonably well with published dislocation dynamics simulations [36] as shown in Fig. 6. As in Barton et al. [16], use of anisotropic elasticity in the crystal plasticity model requires comparing the plastic strains in the dislocation dynamics simulations with those in our crystal plasticity simulations. Our model relies on a scalar form of SIA loop evolution, while Barton et al. employ a tensorial form of the SIA density rate to account for the 3-D nature 
Copyright Table 3: Values of the parameters used in the CDD crystal plasticity model in the SIA loop terms, Eqns. 23 and 21 , for verification of the SIA loop following Barton et al. [16]

\begin{tabular}{ccl}
\hline Parameter & Value & Description \\
\hline$\alpha_{S I A}$ & 0.7 & Hardening coeff. [37] \\
$\beta_{S I A}$ & 1.0 & Annihilation coeff. [32] \\
$R_{S I A}$ & $15 b \mathrm{~mm}$ & Annihilation radius [32] \\
$d_{S I A}$ & $2.48 \times 10^{-5} \mathrm{~mm}$ & Average diameter [16] \\
\hline
\end{tabular}

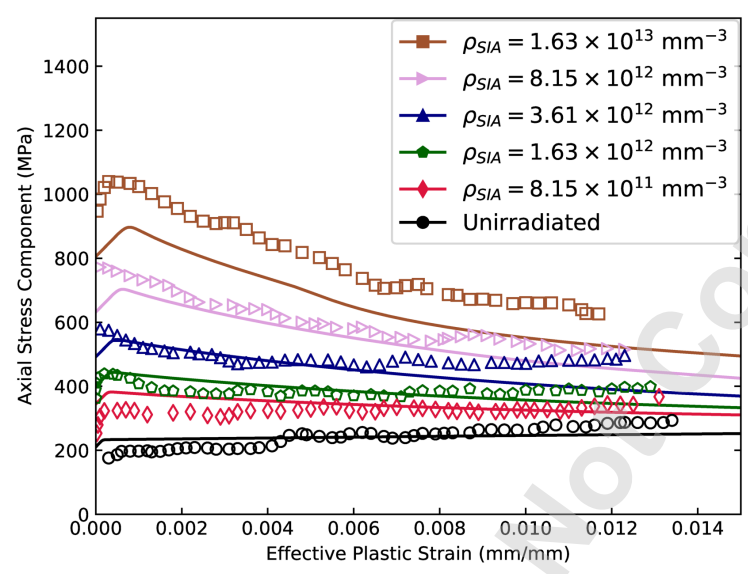

Fig. 6: Comparison of crystal plasticity simulations with dislocation dynamics simulations of the stress-plastic strain evolution, with the varying initial values for SIA loop densities demonstrating trends similar to those in [36] particularly at the lower initial SIA loop densities. Here we focus on the alignment of the trends given the underlying differences in the discrete dislocation dynamics and continuum-level crystal plasticity frameworks, limited by the strain values from dislocation dynamics simulations. The dislocation dynamics data (represented with hollow markers) are reproduced from [16]; our crystal plasticity simulation results are represented by the solid lines.

of SIA loop interaction [16]. The 3-D interaction among the SIA loops and the dislocations is accounted for in our model with a cube root term, Eqn. 21. Comparison of this crystal plasticity model with the dislocation dynamics simulation results [16] (Fig. 6) demonstrates alignment of the trends. The misalignment between the model predictions is due in part to difference in the discrete and continuum approaches of the dislocation dynamics and crystal plasticity frameworks, respectively, with the continuum crystal plasticity method demonstrating less variability than the discrete dislocation dynamics model results. Based on these results, our scalar SIA loop density evolution rate model, Eqn. 23, can acceptably replicate the lower length scale trends for varying initial SIA loop densities in a less computationally intensive manner.

\subsection{Polycrystalline Simulations of Irradiated $\alpha$-Iron}

We compare the results of our CDD crystal plasticity model to irradiated polycrystalline experimental data in order to evaluate the model's ability to predict RPV behavior after exposure to radiation. As a first step, we compared a polycrystalline application of the CDD crystal plasticity model to experimental data on unirradiated $\alpha$-iron. Our simplified polycrystalline geometry consisted of 27 equally sized cubic grains with a diameter of $250 \times 10^{-6} \mathrm{~mm}$ [38]. Each grain was meshed with 216 
Copyright Table 4: balues of the parameters used in the SIA loop terms of the crystal plasticity model for polycrystalline $\alpha$-iron exposed to a radiation dose of $0.1 \mathrm{dpa}$, including initial conditions

\begin{tabular}{ccl}
\hline Parameter & Value & Description \\
\hline$\alpha_{S I A}$ & 0.6 & Hardening coeff. \\
$\beta_{S I A}$ & 0.01 & Loop annihilation coeff. \\
$R_{S I A}$ & $15 \mathrm{bm}$ & Annihilation radius [32] \\
$\rho_{S I A}$ & $1.2 \times 10^{12} \mathrm{~mm}^{-3}$ & Initial density [40] \\
$d_{S I A}$ & $7.0 \times 10^{-6} \mathrm{~mm}$ & Average diameter [40] \\
\hline
\end{tabular}

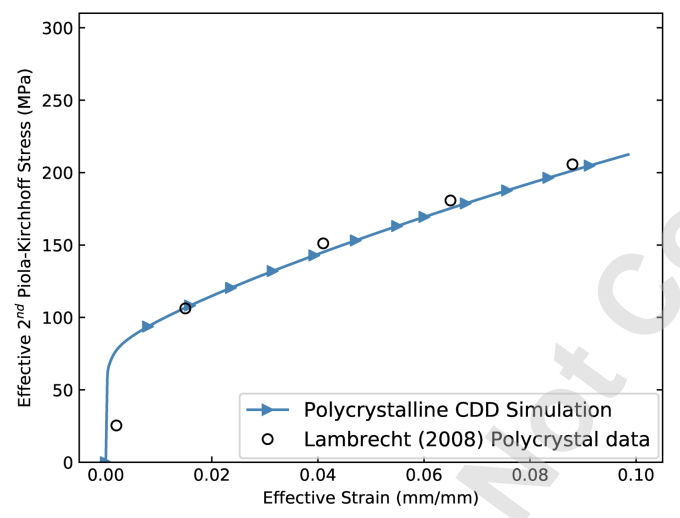

(a) CDD model predictions for unirradiated polycrystalline $\alpha$-iron

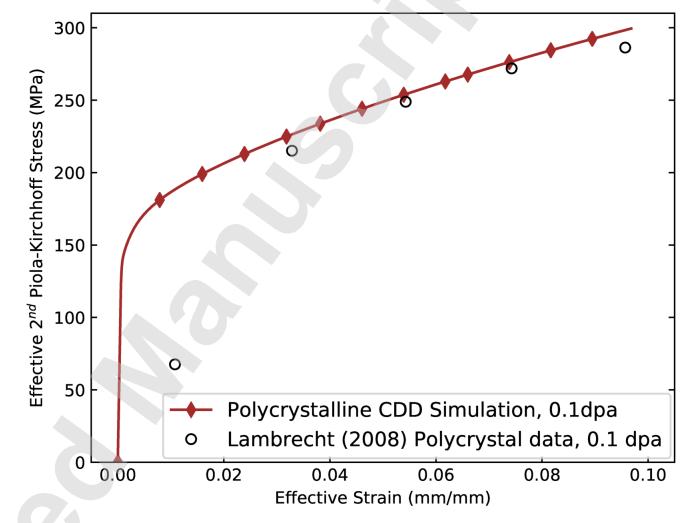

(b) CDD model predictions for radiated (0.1dpa) polycrystalline $\alpha$-iron

Fig. 7: Results of the CDD model applied to a simplified cubic polycrystalline geometry captures the stress-strain response of unirradiated and radiated $(0.1 \mathrm{dpa}) \alpha$-iron. The experimental data points for $\alpha$-iron are reproduced from Lambrecht et al. [38].

elements, for a total of 5832 8-node hexahedron elements in the full mesh. The orientations of the grains were determined through random assignment of the three Bunge Euler angles, within the usual angle bounds, using the Python random number generator with a normal distribution [39].

The dislocation evolution parameters from Tab. 2 for the [100] loading orientation were used in these polycrystalline simulations. The same $\alpha$-iron material parameters from Tab. 1, except for the Peierls stress value: here we used the 
Copyright polycrystaliine value of $11 \mathrm{MPa}$ [23]. We applied an initial dislocation density value of $5 \times 10^{7} \mathrm{~mm}^{-2}$, which falls within the experimentally measured $7 \pm 2 \times 10^{7} \mathrm{~mm}^{-2}$ range [38]; this value of initial dislocation density was selected by calibrating to the unirradiated data for polycrystalline $\alpha$-iron. The initial dislocation density value was split evenly between the mobile and immobile initial dislocation densities. We applied the symmetry boundary condition to the model, with a displacement loading rate corresponding to the strain rate of $2 \times 10^{-4} 1 / \mathrm{s}$ in order to match the experimental loading conditions.

The same mesh, Bunge Euler angles, and parameters were retained for the irradiated polycrystalline simulation. To capture the effect of the irradiation defects, we included the terms for the SIA loop evolution, Eqn. 23, and interaction with the dislocations, Eqn. 21. The parameters for these equations, selected to correspond to an irradiation dose of 0.1 dpa, are given in Tab. 4. We set the value of the dislocation slip-system hardening coefficient, $\alpha_{S I A}$, to 0.6 , which lies between the values of 0.7 and 0.37 used in other studies on irradiated $\alpha$-iron [17,37]. Following the approaches of Li et al. and Chakraborty and Biner, we also lowered the value of the SIA loop annihilation coefficient by a factor of 100 [17,28]; we hypothesize that this reduction of the SIA loop annihilation constant is a result of greatly reducing the loop diameter from that used in the comparison with the dislocation dynamics simulations in Section 4.3.

The stress response of the cubic polycrstalline simulation is calculated as an effective von Mises-type stress measure, equally averaged across all quadrature points in the mesh. In Fig. 7 this effective second Piola-Kirchhoff stress measure is plotted against an effective strain, then compared to the experimental data. The CDD crystal plasticity simulations agree with the unirradiated experiment and irradiated experimental data trends. The CDD simulation results' slight underprediction of the experimentally measured data in Fig. 7 indicates that the CDD crystal plasticity model lacks a hardening contribution. A possible correction to the underpredicted hardening is a strain-gradient-type term. Such a term is often used in crystal plasticity frameworks to capture the effects of grain boundaries on the stress response of the bulk crystal [22].

The general alignment of our CDD model with the measured polycrystalline response-even when applied to a simplistic cubic geometry-demonstrates the capability of our crystal plasticity model to predict the hardening behavior of $\alpha$-iron exposed to irradiation. The physically based dislocation and dislocation-SIA loop interaction terms of the CDD crystal plasticity model can successfully capture the complex physical mechanisms of irradiated $\alpha$-iron.

\section{Conclusions}

We have developed and applied a CDD crystal plasticity model with dislocation evolution terms based in physical dislocation interaction mechanisms. The dislocation evolution is coupled with the SIA loop evolution in acknowledgement of the interstitial loops' significant impact on the irradiation behavior of RPV steels. This model leverages the results of lower length scale molecular dynamics and dislocation dynamics simulations to establish evolution equations for both the dislocations and SIA loops. We calibrated the dislocation evolution components of the crystal plasticity model against single-crystal $\alpha$-iron tensile experiments.

The importance of the stochastic dislocation cross slip model in conjunction with the anisotropic strength of the BCC slip systems is necessary to correctly capture the dislocation behavior in a loading orientation selected for single slip system activation. The anisotropy correction factor on the $\{112\}$ type slip systems allows these slip systems to absorb dislocations, 
Copyright without increasing their own mobile dislocation densities, from active lower length $\{111\}$ type slip systems. The transfer of dislocations through cross slip from softer to harder slip systems prevents overhardening of the single-crystal response.

Verification of the coupling between the dislocation evolution and the SIA loop evolution was performed by comparing the simulation trends from this model with trends from dislocation dynamics simulations. We then applied the CDD crystal plasticity model to a polycrystalline application with a simplistic geometry. The CDD model demonstrates the capability to predict the stress response of irradiated polycrystalline $\alpha$-iron, and the results of this mechanism-based CDD crystal plasticity model can be used to inform engineering scale models that rely on the stress response from an evolving microstructure under radiation and deformation conditions.

\section{Acknowledgements}

This manuscript includes work performed by some of the authors while contractors of the U.S. Government under Contract DE-AC07-05ID14517. Accordingly, the U.S. Government retains a non-exclusive, royalty free license to publish or reproduce the published form of this contribution, or allow others to do so, for U.S. Government purposes. This research made use of the resources of the High Performance Computing Center at Idaho National Laboratory. 
[1] Allen, T. R., and Busby, J. T., 2009. "Radiation damage concerns for extended light water reactor service”. JOM, 61(7), pp. 29-34.

[2] Zinkle, S. J., and Ghoniem, N. M., 2011. "Prospects for accelerated development of high performance structural materials". Journal of Nuclear Materials, 417(1-3), pp. 2-8.

[3] Singh, B., Foreman, A., and Trinkaus, H., 1997. "Radiation hardening revisited: role of intracascade clustering". Journal of Nuclear Materials, 249(2-3), pp. 103-115.

[4] Zinkle, S. J., and Matsukawa, Y., 2004. “Observation and analysis of defect cluster production and interactions with dislocations". Journal of Nuclear Materials, 329, pp. 88-96.

[5] de la Rubia, T., Zbib, H., Khraishi, T., Wirth, B., Victoria, M., and Caturla, M., 2000. "Multiscale modelling of plastic flow localization in irradiated materials". Nature, 406(6798), pp. 871-4.

[6] Klueh, R., and Nelson, A., 2007. "Ferritic/martensitic steels for next-generation reactors". Journal of Nuclear Materials, 371(1), pp. 37-52. Nuclear Fuels and Structural Materials 1.

[7] Arsenlis, A., and Parks, D. M., 2002. "Modeling the evolution of crystallographic dislocation density in crystal plasticity”. Journal of the Mechanics and Physics of Solids, 50(9), pp. 1979-2009.

[8] Cheong, K.-S., and Busso, E. P., 2004. "Discrete dislocation density modelling of single phase FCC polycrystal aggregates". Acta Materialia, 52(19), pp. 5665-5675.

[9] Kocks, U., 1976. “Laws for work-hardening and low-temperature creep”. J. Eng. Mater., 98(1), pp. 76-85.

[10] Roters, F., Raabe, D., and Gottstein, G., 2000. "Work hardening in heterogeneous alloys — a microstructural approach based on three internal state variables". Acta Materialia, 48(17), pp. 4181-4189.

[11] Ma, A., and Roters, F., 2004. "A constitutive model for FCC single crystals based on dislocation densities and its application to uniaxial compression of aluminium single crystals”. Acta Materialia, 52(12), pp. 3603-3612.

[12] Arsenlis, A., Wirth, B. D., and Rhee, M., 2004. "Dislocation density-based constitutive model for the mechanical behaviour of irradiated Cu”. Philosophical Magazine, 84(34), pp. 3617-3635.

[13] Wirth, B. D., Caturla, M. J., Diaz de la Rubia, T., Khraishi, T., and Zbib, H., 2001. "Mechanical property degradation in irradiated materials: A multiscale modeling approach". Nuclear Instruments and Methods in Physics Research, Section B: Beam Interactions with Materials and Atoms, 180(1-4), pp. 23-31.

[14] L. Vincent, M. Libert, B. Marini, C., 2010. “Towards a modelling of RPV steel brittle fracture using crystal plasticity computation on polycrystalline aggregates”. Journal of Nuclear Materials, 406, pp. 91-96.

[15] Patra, A., and McDowell, D. L., 2013. "Continuum modeling of localized deformation in irradiated BCC materials". Journal of Nuclear Materials, 432(1), pp. 414-427.

[16] Barton, N. R., Arsenlis, A., and Marian, J., 2013. “A polycrystal plasticity model of strain localization in irradiated iron". Journal of the Mechanics and Physics of Solids, 61(2), pp. 341-351.

[17] Chakraborty, P., and Biner, S. B., 2016. "Crystal plasticity modeling of irradiation effects on flow stress in pure-iron and iron-copper alloys". Mechanics of Materials, 101, pp. 71-80. 
[19] Orowan, E., 1940. "Problems of plastic gliding". Proceedings of the Physical Society, 52(1), p. 8.

[20] Asaro, R. J., and Needleman, A., 1985. "Overview no. 42: Texture development and strain hardening in rate dependent polycrystals". Acta metallurgica, 33(6), pp. 923-953.

[21] Ohashi, T., 1994. "Numerical modelling of plastic multislip in metal crystals of FCC type". Philosophical Magazine A, 70(5), pp. 793-803.

[22] Roters, F., Eisenlohr, P., Hantcherli, L., Tjahjanto, D. D., Bieler, T. R., and Raabe, D., 2010. “Overview of constitutive laws, kinematics, homogenization and multiscale methods in crystal plasticity finite-element modeling: Theory, experiments, applications". Acta Materialia, 58(4), pp. 1152-1211.

[23] Hirth, J. P., and Lothe, J., 1982. Theory of Dislocations. John Wiley \& Sons.

[24] Zbib, H. M., Díaz De La Rubia, T., Rhee, M., and P. Hirth, J., 2000. “3D dislocation dynamics: Stress-strain behavior and hardening mechanisms in FCC and BCC metals". Journal of Nuclear Materials, 276(1), pp. 154-165.

[25] Lyu, H., Ruimi, A., and Zbib, H. M., 2015. "A dislocation-based model for deformation and size effect in multi-phase steels". International Journal of Plasticity, 72, pp. 44 - 59.

[26] Raabe, D., 1995. "Simulation of rolling textures of BCC metals considering grain interactions and crystallographic slip on $\{110\},\{112\}$ and $\{123\}$ planes". Materials Science and Engineering: A, 197(1), pp. 31-37.

[27] Alankar, A., Field, D. P., and Raabe, D., 2014. "Plastic anisotropy of electro-deposited pure $\alpha$-iron with sharp crystallographic $<111>$ texture in normal direction: Analysis by an explicitly dislocation-based crystal plasticity model". International Journal of Plasticity, 52, pp. 18-32.

[28] Li, D., Zbib, H., Sun, X., and Khaleel, M., 2014. "Predicting plastic flow and irradiation hardening of iron single crystal with mechanism-based continuum dislocation dynamics”. International Journal of Plasticity, 52, pp. 3 - 17.

[29] Taheri-Nassaj, N., and Zbib, H. M., 2016. "A mesoscale model of plasticity: Dislocation dynamics and patterning (one-dimensional)". J. Eng. Mater., 138(4), p. 041015.

[30] Püschl, W., 2002. "Models for dislocation cross-slip in close-packed crystal structures: a critical review". Progress in Materials Science, 47(4), pp. 415 - 461.

[31] Rhee, M., Zbib, H. M., Hirth, J. P., Huang, H., and de la Rubia, T., 1998. "Models for long-/short-range interactions and cross slip in 3d dislocation simulation of bcc single crystals”. Modelling and Simulation in Materials Science and Engineering, 6(4), p. 467.

[32] Mastorakos, I., and Zbib, H., 2014. "A multiscale approach to study the effect of chromium and nickel concentration in the hardening of iron alloys". Journal of Nuclear Materials, 449(1), pp. 101-110.

[33] Permann, C. J., Gaston, D. R., Andrš, D., Carlsen, R. W., Kong, F., Lindsay, A. D., Miller, J. M., Peterson, J. W., Slaughter, A. E., Stogner, R. H., and Martineau, R. C., 2020. "MOOSE: Enabling massively parallel multiphysics simulation”. SoftwareX, 11, p. 100430.

[34] Keh, A., 1965. "Work hardening and deformation sub-structure in iron single crystals deformed in tension at 298 K". Philosophical Magazine, 12(115), pp. 9-30. 
Copyright 35] Lee, M., Lim, H., Adams, B., Hirth, J., and Wagoner, R., 2010. "A dislocation density-based single crystal constitutive equation”. International Journal of Plasticity, 26(7), pp. 925-938.

[36] Arsenlis, A., Rhee, M., Hommes, G., Cook, R., and Marian, J., 2012. “A dislocation dynamics study of the transition from homogeneous to heterogeneous deformation in irradiated body-centered cubic iron". Acta Materialia, 60(9), pp. $3748-3757$.

[37] Lambrecht, M., Meslin, E., Malerba, L., Hernández-Mayoral, M., Bergner, F., Pareige, P., Radiguet, B., and Almazouzi, A., 2010. "On the correlation between irradiation-induced microstructural features and the hardening of reactor pressure vessel steels". Journal of Nuclear Materials, 406(1), pp. 84-89.

[38] Lambrecht, M., Malerba, L., and Almazouzi, A., 2008. "Influence of different chemical elements on irradiation-induced hardening embrittlement of RPV steels”. Journal of Nuclear Materials, 378(3), pp. 282-290.

[39] Python Software Foundation, 2018. The python standard library. https://www.python.org/. Accessed: 2018-07-18.

[40] Meslin, E., Lambrecht, M., Hernández-Mayoral, M., Bergner, F., Malerba, L., Pareige, P., Radiguet, B., Barbu, A., Gómez-Briceño, D., Ulbricht, A., and Almazouzi, A., 2010. "Characterization of neutron-irradiated ferritic model alloys and a RPV steel from combined APT, SANS, TEM and PAS analyses”. Journal of Nuclear Materials, 406(1), pp. 73-83. 
$1 \quad$ Values of the parameters used in the crystal plasticity model for single-crystal $\alpha$-iron simulations . . . . . .

2 Dislocation evolution parameters for Eqns. 9 and 10, calibrated for the crystal plasticity model in singlecrystal $\alpha$-iron simulations for three different loading directions . . . . . . . . . . . . . .

3 Values of the parameters used in the CDD crystal plasticity model in the SIA loop terms, Eqns. 23 and 21 , for verification of the SIA loop following Barton et al. [16] . . . . . . . . . . . . . . . . .

$4 \quad$ Values of the parameters used in the SIA loop terms of the crystal plasticity model for polycrystalline $\alpha$-iron exposed to a radiation dose of $0.1 \mathrm{dpa}$, including initial conditions $\ldots \ldots \ldots$ 


\section{Copyright fist or Figures}

1 Verification of the dislocation mechanism term coefficients in Eqns. 11-16 against single-crystal BCC $\alpha$-iron in three loading direction [34], reproduced from Lee et al. [35]. The misalignment at low strains of the CDD model with the experiment data points in the [01̄1] and [ $\overline{3} 48]$ loading directions is a consequence of the weight given to the [100] loading direction in setting the uniform values for the parameters $\alpha_{1}-\alpha_{4}$ and $\alpha_{6}$ across all three loading directions. $\ldots \ldots \ldots \ldots \ldots \ldots \ldots \ldots \ldots$

2 Probability of dislocation cross slip on slip systems with an applied shear stress above the initial lattice friction strength, as calculated directly from Eqn. 18, before normalization. The stochastic-PM approach demonstrates the saturation trends shown in the mobile dislocation evolution, Fig. 4a, while the stochasticMC approach demonstrates increased probability with increasing strain, Fig. 4 b. . . . . . . . . . .

3 Evolution of the cross slip dislocation density. Note that the $(\overline{2} 11)[111]$ system is expected to be the favored active slip system under the [348] loading direction [34]. The value of the $\alpha_{5}$ coefficient in the stochasticPM approach was adjusted to create parity the in $(\overline{2} 11)[111]$ cross slip dislocation at the beginning of the simulation, although the mobile dislocation evolution, Fig. 4, impacts the available dislocations density to

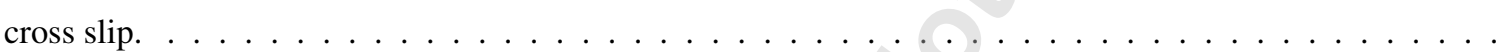

4 Evolution of the mobile dislocation density on select slip systems, with the (211)[111] system expected to be the favored active slip system [34]. The stochastic-PM approach demonstrates saturation of the mobile dislocation densities; in contrast, the stochastic-MC cross slip approach is able to maintain dislocation density growth on this primary slip system. Although other slip systems demonstrate growth as a result of slip system activation, the $(\overline{2} 11)[111]$ system shows the highest density growth in accordance with its role as the primary slip system.

5 The comparison of the cross slip probability among the six slip systems in the [111] cross slip family demonstrates that the (211)[111] and (101)[111] systems have similar probabilities as the strain increases. Using the stochastic-MC approach, the slip system evolution demonstrates the influence of the anistropy correction for the intrinsic lattice friction strength, Eqn. 7. This anisotropy correction hardens the $\{110\}$-type systems more than it does the $\{112\}$-type systems, enabling dislocations to cross slip from the lower slip resistance systems to the higher ones, without additional slip system activation. . . . . . . . . . . . . .

6 Comparison of crystal plasticity simulations with dislocation dynamics simulations of the stress-plastic strain evolution, with the varying initial values for SIA loop densities demonstrating trends similar to those in [36] particularly at the lower initial SIA loop densities. Here we focus on the alignment of the trends given the underlying differences in the discrete dislocation dynamics and continuum-level crystal plasticity frameworks, limited by the strain values from dislocation dynamics simulations. The dislocation dynamics data (represented with hollow markers) are reproduced from [16]; our crystal plasticity simulation results are

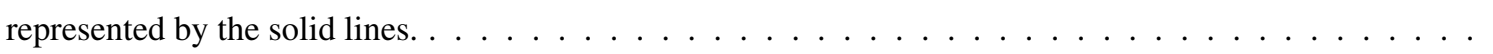



response of unirradiated and radiated $(0.1 \mathrm{dpa}) \alpha$-iron. The experimental data points for $\alpha$-iron are reproduced from Lambrecht et al. $[38] \ldots \ldots \ldots \ldots \ldots \ldots \ldots \ldots \ldots \ldots \ldots$ 
Table 1: Values of the parameters used in the crystal plasticity model for single-crystal $\alpha$-iron simulations

\begin{tabular}{ccl}
\hline Parameter & Value & Description \\
\hline$C_{11}$ & $242 \times 10^{3} \mathrm{MPa}$ & Elastic constant [23] \\
$C_{12}$ & $150 \times 10^{3} \mathrm{MPa}$ & Elastic constant [23] \\
$C_{44}$ & $112 \times 10^{3} \mathrm{MPa}$ & Elastic constant [23] \\
$\mu$ & $80 \times 10^{3} \mathrm{MPa}$ & Shear modulus [23] \\
$b$ & $2.48 \times 10^{-7} \mathrm{~mm}$ & Burgers vector [23] \\
$\dot{\gamma}_{o}$ & $4.0 \times 10^{-2}$ & Reference strain rate \\
$m$ & 0.012 & Strain rate exponent \\
$\alpha_{m b h}$ & 0.4 & Dispersed barrier \\
$\Omega^{\alpha \alpha}$ & 1.0 & Self-hardening \\
$\Omega^{\alpha \beta}$ & 0.2 & Latent-hardening \\
$R_{c}$ & $15 b \mathrm{~mm}$ & Radius of capture \\
$\tau^{*}$ & $4 \times 10^{-3} \cdot \mu$ & Critical cross slip stress \\
$V_{a}$ & $20 b^{3}$ & Cross slip volume \\
$k$ & $1.38065 \times 10^{-20}$ & Bolztmann constant \\
$T$ & $298 \mathrm{~K}$ & Temperature \\
\hline
\end{tabular}


Table 2: Dislocation evolution parameters for Eqns. 9 and 10, calibrated for the crystal plasticity model in single-crystal $\alpha$-iron simulations for three different loading directions

\begin{tabular}{llll}
\hline \multicolumn{4}{c}{ Loading Direction } \\
& {$[100]$} & {$[0 \overline{1} \overline{1}]$} & {$[\overline{3} 48]$} \\
\hline$\alpha_{1}$ & 0.03 & 0.03 & 0.03 \\
$\alpha_{2}$ & 0.5 & 0.5 & 0.5 \\
$\alpha_{3}$ & 0.002 & 0.002 & 0.002 \\
$\alpha_{4}$ & 0.002 & 0.002 & 0.002 \\
$\alpha_{5}$ & 0.015 & 0.0335 & 0.044 \\
$\alpha_{6}$ & 1.0 & 1.0 & 1.0 \\
$g_{o}$ & $15 \mathrm{MPa}$ & $8.8 \mathrm{MPa}$ & $8.8 \mathrm{MPa}$ \\
\hline
\end{tabular}


Table 3: Values of the parameters used in the CDD crystal plasticity model in the SIA loop terms, Eqns. 23 and 21 , for verification of the SIA loop following Barton et al. [16]

\begin{tabular}{ccl}
\hline Parameter & Value & Description \\
\hline$\alpha_{S I A}$ & 0.7 & Hardening coeff. [37] \\
$\beta_{S I A}$ & 1.0 & Annihilation coeff. [32] \\
$R_{S I A}$ & $15 b \mathrm{~mm}$ & Annihilation radius [32] \\
$d_{S I A}$ & $2.48 \times 10^{-5} \mathrm{~mm}$ & Average diameter [16] \\
\hline
\end{tabular}


Table 4: Values of the parameters used in the SIA loop terms of the crystal plasticity model for polycrystalline $\alpha$-iron exposed to a radiation dose of $0.1 \mathrm{dpa}$, including initial conditions

\begin{tabular}{ccl}
\hline Parameter & Value & Description \\
\hline$\alpha_{S I A}$ & 0.6 & Hardening coeff. \\
$\beta_{S I A}$ & 0.01 & Loop annihilation coeff. \\
$R_{S I A}$ & $15 \mathrm{bm}$ & Annihilation radius [32] \\
$\rho_{S I A}$ & $1.2 \times 10^{12} \mathrm{~mm}^{-3}$ & Initial density [40] \\
$d_{S I A}$ & $7.0 \times 10^{-6} \mathrm{~mm}$ & Average diameter [40] \\
\hline
\end{tabular}




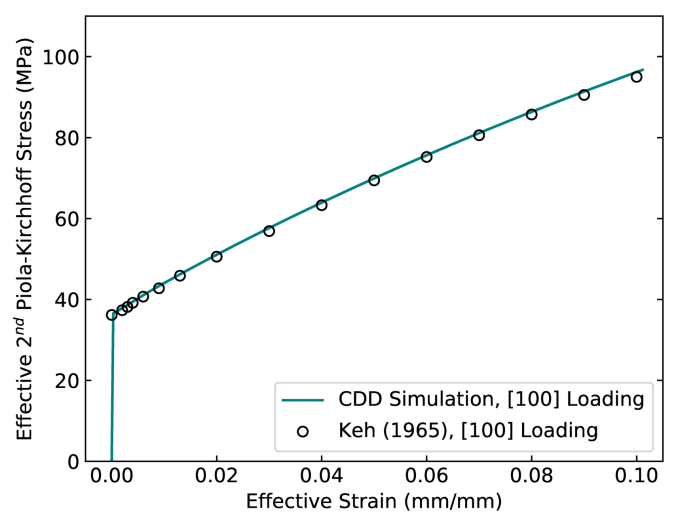

(a) Verification in the [100] loading direction of the dislocation-mechanism term $\alpha$ coefficients against single-crystal BCC $\alpha$-iron

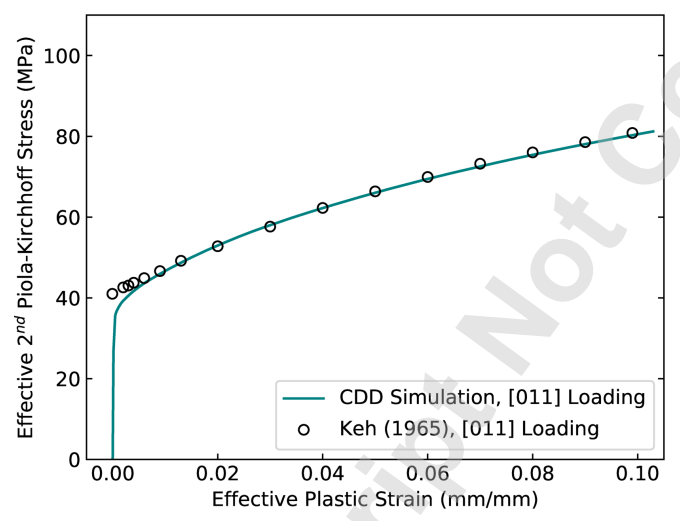

(b) Comparison of the CDD model in the [01̄1] loading direction using the $\alpha$ values given in Tab. 2 with singlecrystal BCC $\alpha$-iron

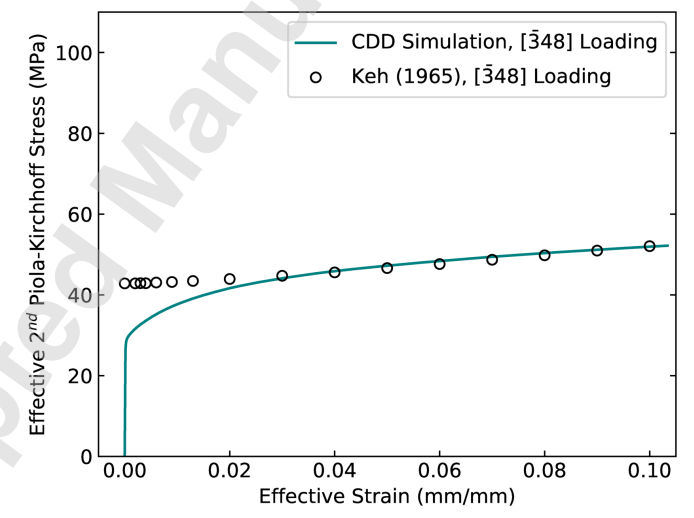

(c) Demonstration in the [3 348$]$ loading direction of the dislocation evolution mechanisms $\alpha$ coefficients against single-crystal BCC $\alpha$-iron

Fig. 1: Verification of the dislocation mechanism term coefficients in Eqns. 11-16 against single-crystal BCC $\alpha$-iron in three loading direction [34], reproduced from Lee et al. [35]. The misalignment at low strains of the CDD model with the

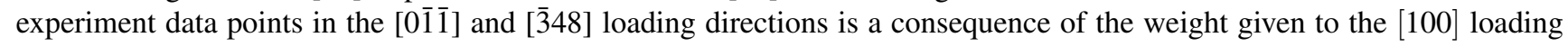
direction in setting the uniform values for the parameters $\alpha_{1}-\alpha_{4}$ and $\alpha_{6}$ across all three loading directions. 


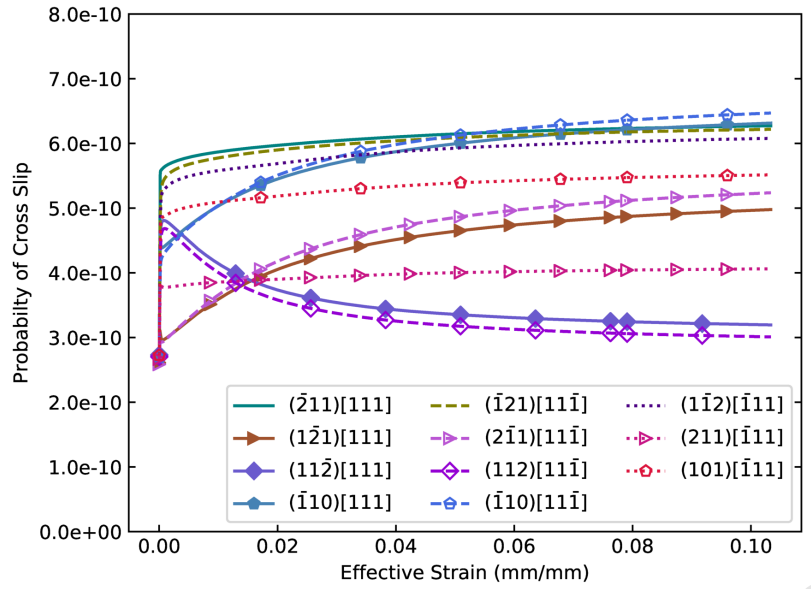

(a) Stochastic-PM approach

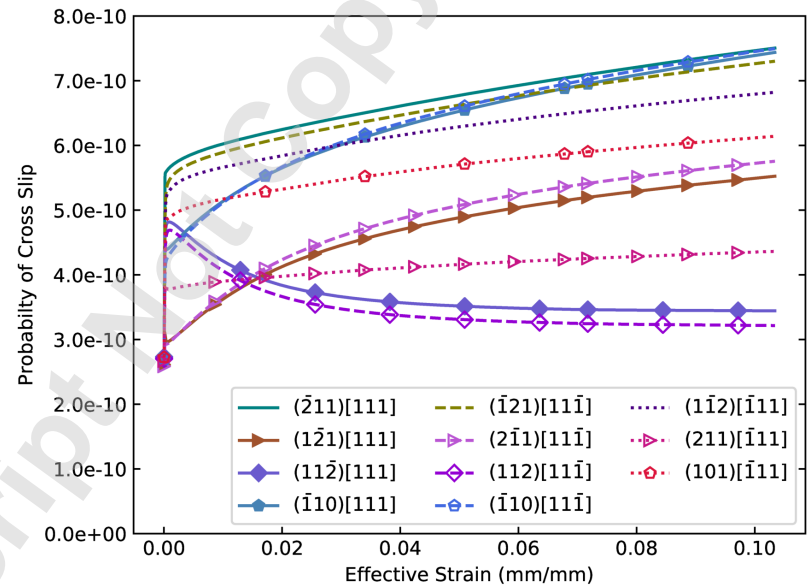

(b) Stochastic-MC approach

Fig. 2: Probability of dislocation cross slip on slip systems with an applied shear stress above the initial lattice friction strength, as calculated directly from Eqn. 18, before normalization. The stochastic-PM approach demonstrates the saturation trends shown in the mobile dislocation evolution, Fig. 4a, while the stochastic-MC approach demonstrates increased probability with increasing strain, Fig. 4 b. 


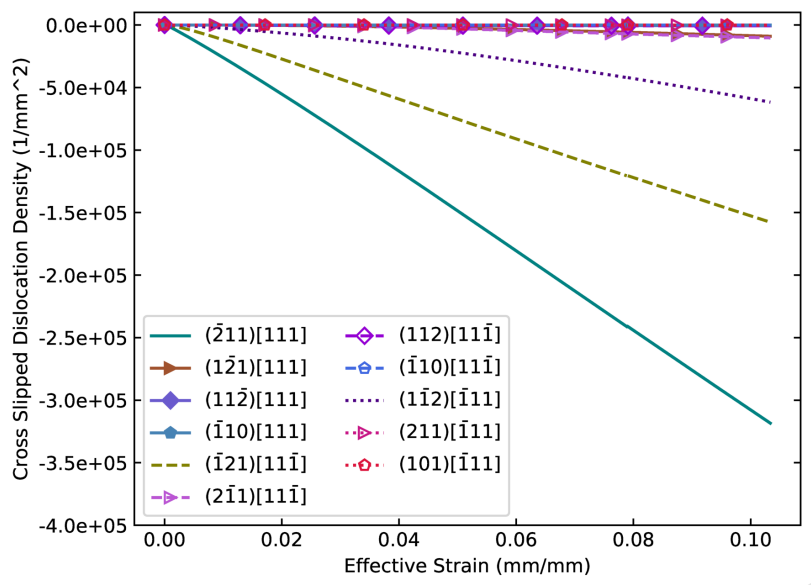

(a) Stochastic-PM approach from Eqn. 19

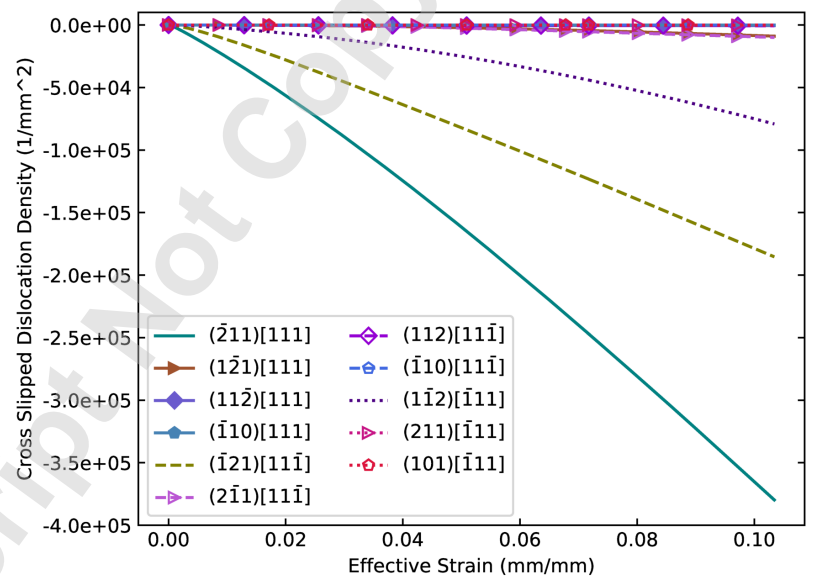

(b) Stochastic-MC approach, as calculated with Eqn. 20

Fig. 3: Evolution of the cross slip dislocation density. Note that the $(\overline{2} 11)[111]$ system is expected to be the favored active slip system under the [348] loading direction [34]. The value of the $\alpha_{5}$ coefficient in the stochastic-PM approach was adjusted to create parity the in $(\overline{2} 11)$ [111] cross slip dislocation at the beginning of the simulation, although the mobile dislocation evolution, Fig. 4, impacts the available dislocations density to cross slip. 


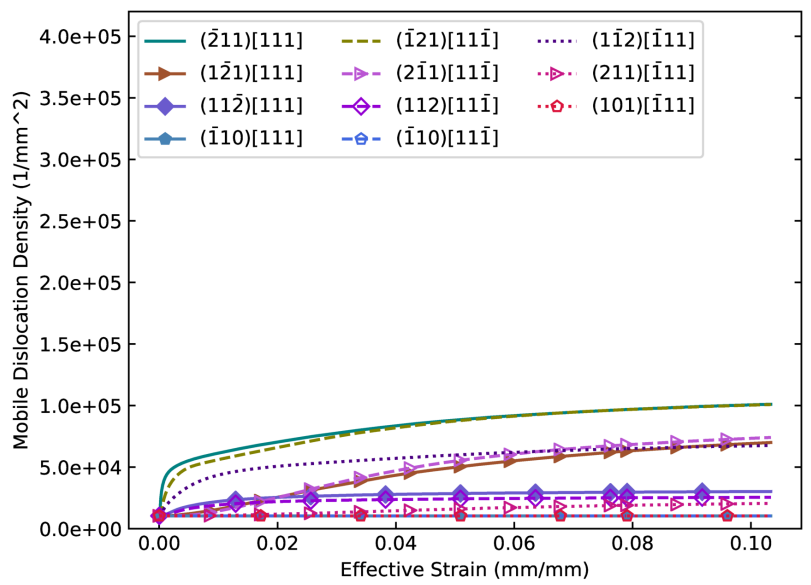

(a) The stochastic-PM approach demonstrates saturation

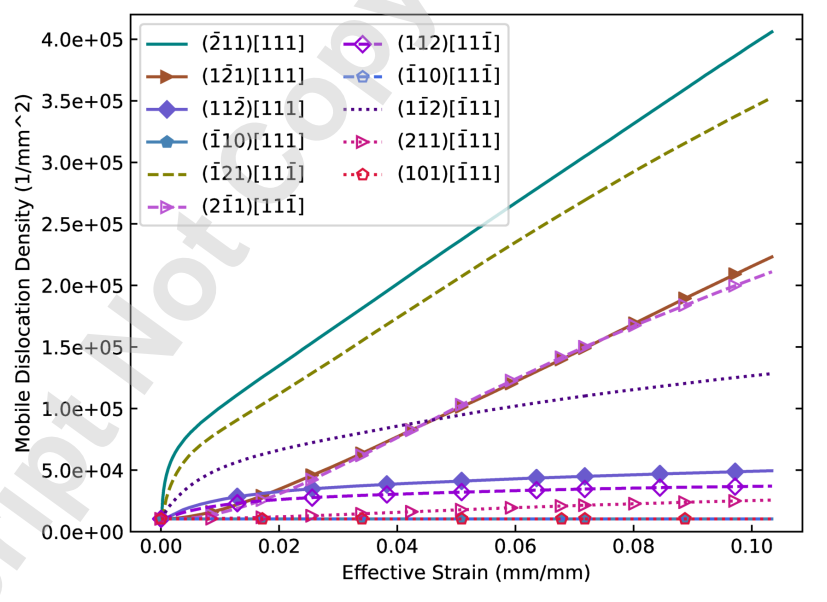

(b) The stochastic-MC approach demonstrates growth

Fig. 4: Evolution of the mobile dislocation density on select slip systems, with the $(\overline{2} 11)[111]$ system expected to be the favored active slip system [34]. The stochastic-PM approach demonstrates saturation of the mobile dislocation densities; in contrast, the stochastic-MC cross slip approach is able to maintain dislocation density growth on this primary slip system. Although other slip systems demonstrate growth as a result of slip system activation, the $(\overline{2} 11)[111]$ system shows the highest density growth in accordance with its role as the primary slip system. 


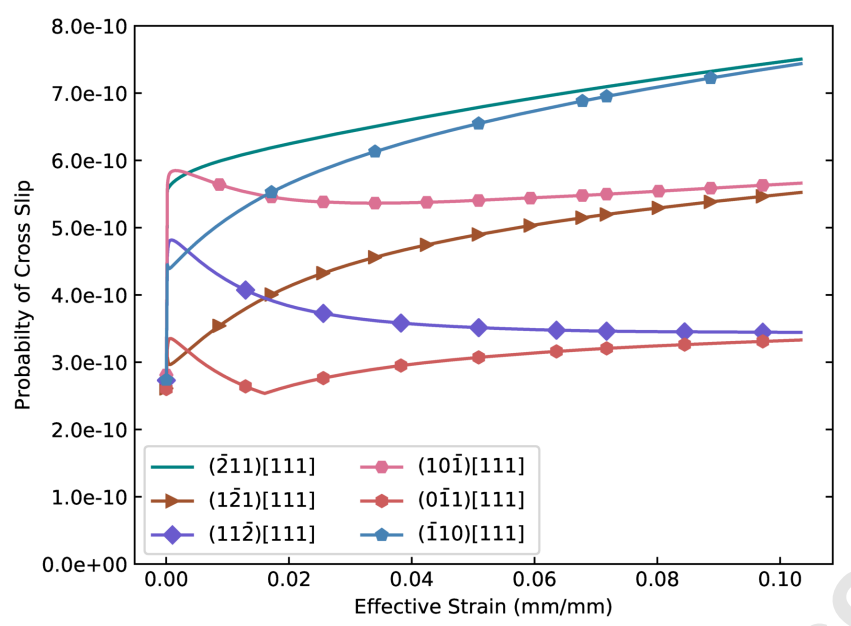

(a) Probability of cross slip in the [111] cross slip family

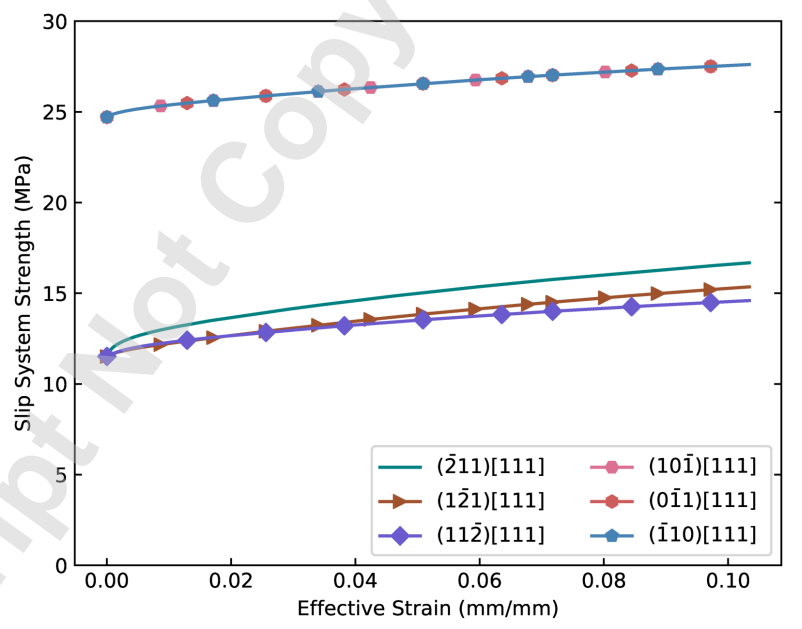

(b) Slip system strength evolution in the [111] cross slip family

Fig. 5: The comparison of the cross slip probability among the six slip systems in the [111] cross slip family demonstrates that the $(\overline{2} 11)[111]$ and (101)[111] systems have similar probabilities as the strain increases. Using the stochastic-MC approach, the slip system evolution demonstrates the influence of the anistropy correction for the intrinsic lattice friction strength, Eqn. 7. This anisotropy correction hardens the $\{110\}$-type systems more than it does the $\{112\}$-type systems, enabling dislocations to cross slip from the lower slip resistance systems to the higher ones, without additional slip system activation. 


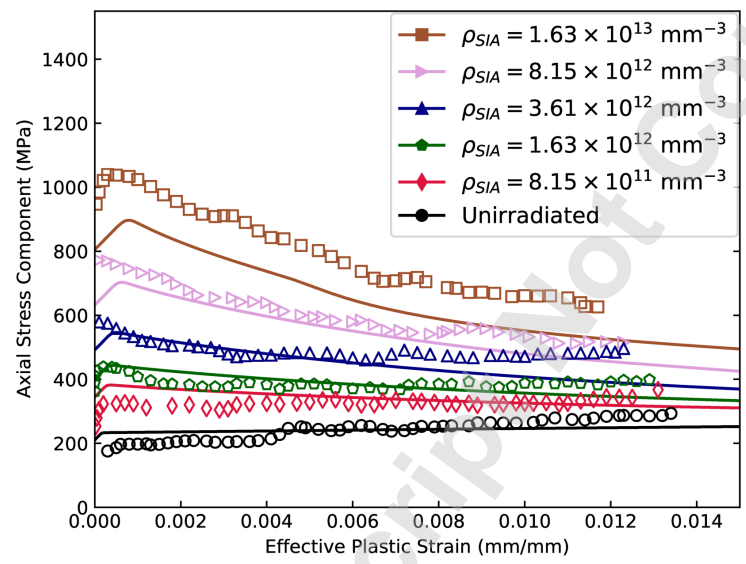

Fig. 6: Comparison of crystal plasticity simulations with dislocation dynamics simulations of the stress-plastic strain evolution, with the varying initial values for SIA loop densities demonstrating trends similar to those in [36] particularly at the lower initial SIA loop densities. Here we focus on the alignment of the trends given the underlying differences in the discrete dislocation dynamics and continuum-level crystal plasticity frameworks, limited by the strain values from dislocation dynamics simulations. The dislocation dynamics data (represented with hollow markers) are reproduced from [16]; our crystal plasticity simulation results are represented by the solid lines. 


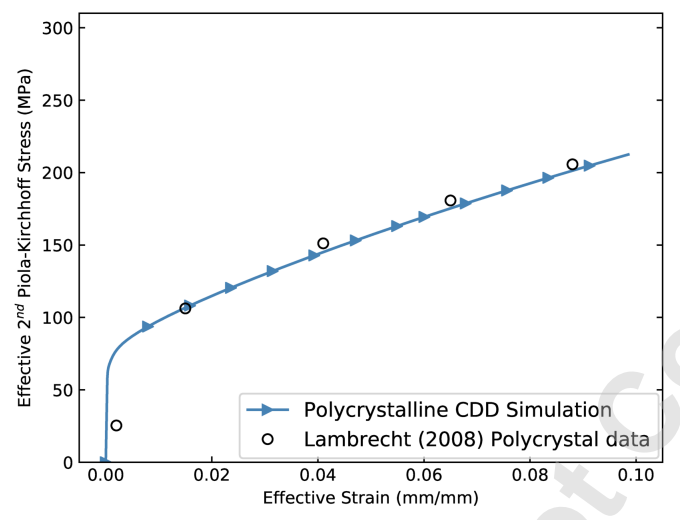

(a) CDD model predictions for unirradiated polycrystalline $\alpha$-iron

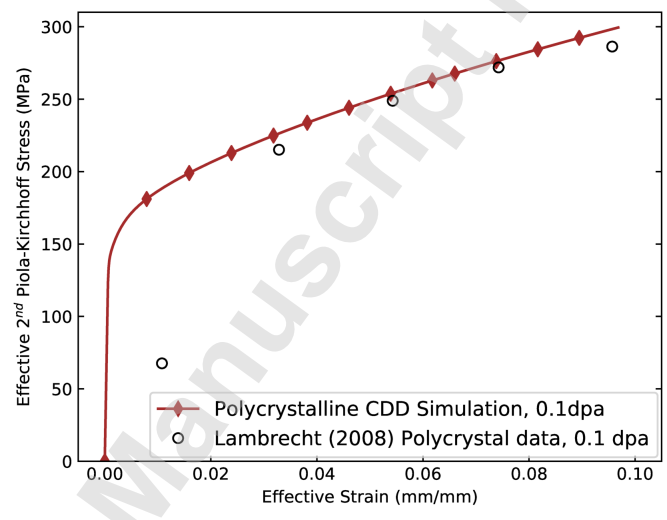

(b) CDD model predictions for radiated (0.1dpa) polycrystalline $\alpha$-iron

Fig. 7: Results of the CDD model applied to a simplified cubic polycrystalline geometry captures the stress-strain response of unirradiated and radiated $(0.1 \mathrm{dpa}) \alpha$-iron. The experimental data points for $\alpha$-iron are reproduced from Lambrecht et al. [38]. 